\title{
Precommitted Investment Strategy versus Time-Consistent Investment Strategy for a General Risk Model with Diffusion
}

\author{
Lidong Zhang, ${ }^{1,2}$ Ximin Rong, ${ }^{3}$ and Ziping $\mathrm{Du}^{4}$ \\ ${ }^{1}$ School of Management, Tianjin University, Tianjin 300072, China \\ ${ }^{2}$ School of Science, Tianjin University of Science and Technology, Tianjin 300457, China \\ ${ }^{3}$ School of Science, Tianjin University, Tianjin 300072, China \\ ${ }^{4}$ College of Economics \& Management, Tianjin University of Science and Technology, Tianjin 300222, China
}

Correspondence should be addressed to Ximin Rong; rongximin@tju.edu.cn

Received 16 February 2014; Accepted 11 March 2014; Published 9 April 2014

Academic Editor: Guanglu Zhou

Copyright (c) 2014 Lidong Zhang et al. This is an open access article distributed under the Creative Commons Attribution License, which permits unrestricted use, distribution, and reproduction in any medium, provided the original work is properly cited.

We mainly study a general risk model and investigate the precommitted strategy and the time-consistent strategy under meanvariance criterion, respectively. A lagrange method is proposed to derive the precommitted investment strategy. Meanwhile from the game theoretical perspective, we find the time-consistent investment strategy by solving the extended Hamilton-Jacobi-Bellman equations. By comparing the precommitted strategy with the time-consistent strategy, we find that the company under the timeconsistent strategy has to give up the better current utility in order to keep a consistent satisfaction over the whole time horizon. Furthermore, we theoretically and numerically provide the effect of the parameters on these two optimal strategies and the corresponding value functions.

\section{Introduction}

There exist two important risk models: the Cramér-Lundberg model (the C-L risk model) and the dual risk model. The C$\mathrm{L}$ risk model describes the surplus process of an insurance company. The insurer has two opposing cash flows: incoming cash premiums and outgoing claims. It can get the premium at a rate $c_{1}>0$ from the insured and pay for claims which arrive according to a Poisson process with intensity $\lambda$ and claims $Z_{1, j}(j=1,2,3, \ldots)$ are independent and identically distributed (i.i.d) nonnegative random variables with mean $\mu_{1}$. The surplus of an insurer which starts with initial surplus $x$ is described as follows:

$$
R_{1}(t)=x+c_{1} t-\sum_{j=1}^{N_{t}} Z_{1, j} \text { for } t \geq 0
$$

Correspondingly, the dual risk model is called dual as opposed to the C-L risk model with applications to insurance.
The dual risk model describes a surplus process of another company engaging in research and development. This company also has two opposing cash flows. The positive incomes or profits arrive according to a Poisson process with intensity $\lambda$ and profits $Z_{2, j}(j=1,2,3, \ldots)$ are i.i.d nonnegative random variables with mean $\mu_{2} . c_{2}$ is the rate of expenses of the company. Thus the surplus of the company is subject to the following equation:

$$
R_{2}(t)=x+\sum_{j=1}^{N(t)} Z_{2, j}-c_{2} t
$$

There are many possible interpretations for the dual model. The surplus can be viewed as the amount of capital of a company (e.g., petroleum or pharmaceutical companies) engaging in research and development where costs are certain and gains are random at random instants. 
By incorporating the C-L risk model and the dual risk model, a general risk model can be given by the following equation:

$$
R(t)=x+\sum_{j=1}^{N(t)} Z_{j}+c t+\sigma_{0} W_{0}(t)
$$

where $c \in \mathbb{R},\{N(t)\}_{t \geq 0}$ is a Poisson process with intensity $\lambda$, and $Z_{j}(j=1,2,3, \ldots)$ are independent and identical double distributed with the probability density function $f_{z}(z)=p \times$ $f_{1}(z) I_{z>0}+(1-p) \times f_{2}(z) I_{z<0}$. The C-L risk model and the dual risk model are all the special examples. This model can also be described as the surplus of a company engaging in research and development where success or failure can cause a greater profit or a bigger loss, respectively.

The literature for mean-variance (MV) analysis of the general risk model has not appeared. Mean-variance analysis for optimal asset allocation is an important result of financial economics. Markowitz [1] proposed the mean-variance approach and it is viewed as the foundation of modern finance theory. Since then, a large number of papers have been published on this topic. The single period case was dealt with by many scholars. The MV optimal portfolio problem in a multiperiod framework and continuous time version is time-inconsistent which means that the Bellman Optimality Principle does not hold.

Nowadays two basic ways are used to deal with timeinconsistency in optimal control problems in the literature. One way is to study the precommitted problem where "optimal" is interpreted as "optimal from the point of view of time zero" and the decision makers themselves follow the policies chosen at the initial time in the future. Zhou and $\mathrm{Li}$ [2] and Li and $\mathrm{Ng}$ [3] made excellent works in dealing with time-inconsistency by employing an embedding technique. Another way is to take the time-inconsistency more seriously and study the problem within a game theoretical framework. One possible interpretation of the time-inconsistency is that our preferences change in a temporally inconsistent way as time goes by, and we can view the MV problem as a game, where the players are the future incarnations of our own preferences. Nash equilibrium points can be found in the game theoretical approach to address general timeinconsistency. See more references in Björk and Murgoci [4], Ekeland and Lazrak [5], and Kryger and Steffensen [6].

Recently many scholars have paid more attention to mean-variance analysis for the risk model. Bäuerle [7] investigated a reinsurance problem and measured deviations from a certain predefined benchmark $b$. Bai and Zhang [8] studied optimal reinsurance-investment (no-shorting) strategy for the mean-variance problem under the following two risk models: a classical risk model and a diffusion risk model. Some other scholars also discussed the reinsuranceinvestment problem from game theoretical framework. Li et al. [9] and Zeng et al [10] investigated the time-consistent investment and reinsurance strategy when the prices of risky assets followed Heston's SV process and a jump diffusion process, respectively.
In this paper, we are concerned with the optimal investment problem for the general risk model under meanvariance criterion. Our study contributes to the literature in three ways. Firstly, we study the general risk model under mean-variance criterion and the precommitted strategy and the time-consistent strategy are derived. Secondly, we propose a simple technique (lagrange technique) to deal with the precommitted investment problem. Our method is different from the lagrange technique proposed by Zhou and Li [2]. They showed that this nonstandard problem (MV) can be embedded into a class of auxiliary stochastic linearquadratic (LQ) problems. The optimal strategy was derived by solving the LQ problem and they calculated the efficient frontier in a closed form for the original portfolio selection. Correspondingly, the precommitted strategy and the efficient frontier are derived together in the process of solving problem (MV) by the lagrange technique we proposed. Thirdly, we investigate the effect of the parameters on the investment strategies and the corresponding value functions from the theoretical and numerical analysis. The comparisons of the value functions and the efficient frontiers show that the company under the time-consistent strategy has to give up the better current utility in order to keep a consistent satisfaction over the whole time horizon.

The rest of this paper is organized as follows. Section 2 describes the model and formulates the problem under meanvariance criterion. In the next two sections, we investigate the precommitted investment strategy and the time-consistent strategy for problem (MV), respectively. In Section 5, numerical analysis is presented for our results.

\section{Problem Formulation}

In this section, we start with a filtered complete probability space $\left(\Omega, \mathscr{F},\left\{\mathscr{F}_{t}\right\}_{0 \leq t \leq T}, \mathscr{P}\right)$, where $T$ represents the time horizon and $\mathscr{F}_{t}$ stands for the information available at time $t$. Here recall the general risk model; namely, the surplus process of a company is given by

$$
R(t)=x+\sum_{j=1}^{N(t)} Z_{j}+c t+\sigma_{0} W_{0}(t)
$$

where $\{N(t)\}_{t \geq 0}$ is a Poisson process with intensity $\lambda, Z_{j}$ is the size of the $j$ th income or profit which is double distributed with the probability density function $f_{z}(z)=p \times f_{1}(z) I_{z>0}+$ $(1-p) \times f_{2}(z) I_{z<0}$ and the first and second moment $\mu_{z}$ and $\sigma_{z}^{2}$, and $W_{0}(t)$ represents the uncertainty of the income. Furthermore assume the expected increase of the surplus per unit time satisfies the positive loading condition, $\lambda \mu_{z}+c>0$.

The financial market consists of a risk-free asset and $n$ risky assets. The company is allowed to invest its surplus into this financial market. The total amount of money invested into $i$ th risky asset at time $t$ is described as $l_{i}(t)$. The price of the risk-free asset $S_{0}$ is subject to the following stochastic differential equation:

$$
d S_{0}(t)=r_{0}(t) S_{0}(t) d t, \quad S_{0}(0)=s_{0}
$$


and the price of the $i$ th risky asset $S_{i}$ satisfies the following stochastic differential equation:

$$
\begin{gathered}
d S_{i}(t)=S_{i}(t)\left(r^{i}(t) d t+\sum_{j=1}^{d} \sigma_{i j}(t) d W_{j}(t)\right), \\
S_{i}(0)=s_{i},
\end{gathered}
$$

where $r_{0}(t)$ is a risk-free rate, $r^{i}(t)>r_{0}(t)$ is the appreciation rate of the $i$ th risky asset and the functions including $r_{0}(t)$ and $r^{i}(t)$, and $\sigma_{i j}(t)$ are all positive continuous bounded functions. $W(t)=\left(W_{1}(t), W_{2}(t), \ldots, W_{d}(t)\right)^{T}$ is a $d$-dimensional standard Brownian motion which is independent of $\sum_{j=1}^{N(t)} Z_{j}$ and $W_{0}(t)$. Here the superscript " $T$ " denotes the transpose of a matrix or a vector and $d \geq n$. Let $X^{l}(t)$ denote the resulting surplus process after incorporating strategy $l$ into (4). The dynamics of $X^{l}(t)$ can be described as follows:

$$
\begin{aligned}
d X^{l}(t)= & \left(r_{0}(t) X^{l}(t)+r^{T}(t) l(t)+c\right) d t \\
& +l^{T}(t) \sigma(t) d W(t)+d \sum_{j=1}^{N(t)} Z_{j}+\sigma_{0} W_{0}(t),
\end{aligned}
$$

where $r(t)=\left(r^{1}(t)-r_{0}(t), r^{2}(t)-r_{0}(t), \ldots, r^{n}(t)-r_{0}(t)\right)^{T}$ and $\sigma(t)=\left(\sigma_{i j}\right)_{n \times d}$. Furthermore, denote $\Sigma(t)=\sigma(t) \sigma^{T}(t)$ and assume that $\Sigma(t)$ is reversible for all $t \in[0, T]$.

For $Q=[0, T] \times \mathbb{R}$, denote $C^{1,2}(Q)=\{\phi(t, x) \mid \phi(t, \cdot)$ is once continuously differentiable on $[0, \mathrm{~T}]$, and $\phi(\cdot, x)$ is twice continuously differentiable on $\mathbb{R}\}$.

For $\phi(t, x) \in C^{1,2}(Q)$, the infinitesimal operator of the surplus process $X^{l}(t)$ is given by the following equation:

$$
\begin{aligned}
\mathscr{A} \phi(t, x)= & \phi_{t}(t, x)+\phi_{x}(t, x)\left(r_{0}(t) x+c+r^{T}(t) l(t)\right) \\
& +\frac{1}{2} \phi_{x x}(t, x)\left(l^{T}(t) \Sigma(t) l(t)+\sigma_{0}^{2}\right) \\
& +\lambda[E \phi(t, x+Z)-\phi(t, x)] .
\end{aligned}
$$

Next, we give the definition of admissible strategy on the general risk process $X^{l}(t)$.

Definition 1 (admissible strategy). A strategy $l=\{l(t)\}_{t \in[0, T]}$ is said to be admissible if it satisfies the following conditions:

(1) $l:[0, \infty] \times \Omega \rightarrow \mathbb{R}$ is an $\mathscr{F}$-adapted process;

(2) $l$ satisfies the integrability condition, $E \int_{0}^{t} l^{T}(t) \Sigma(t)$ $l(t) d s<\infty$ almost surely, for all $t \in[0, T]$;

(3) $\operatorname{SDE}(7)$ has a unique solution corresponding to $l$.

In addition, let $U$ denote the set of all admissible strategies with respect to initial condition $(t, x) \in[0, T] \times \mathbb{R}$. The objective is to find the optimal investment strategy among all the admissible strategies in order to make the expected terminal wealth maximized and the variance of the terminal wealth minimized. The alternative objective is to find a strategy which maximizes the expected terminal wealth minus the variance of the terminal wealth by the biobjective optimization theory. So the objective is changed to find the maximization of the following function:

$$
(\mathrm{MV})\left\{\begin{array}{l}
E_{0, x}\left(X^{l}(T)\right)-\frac{\gamma}{2} \operatorname{Var}_{0, x}\left(X^{l}(T)\right) \longrightarrow \max \\
l \in U
\end{array}\right.
$$

where $\gamma$ is a prespecified risk aversion coefficient, $\operatorname{Var}_{0, x}[\cdot]=$ $\operatorname{Var}\left[\cdot \mid X^{l}(0)=x\right]$. Because this mean-variance criterion lacks the iterated-expectation property, this problem is time-inconsistent in the sense that the Bellman Optimality Principle does not hold. This problem can be reduced to a resolvable problem by virtue of some techniques including the lagrange technique and the game theoretical technique. In the following two sections, optimal investment strategies and the value functions can be explicitly derived in the general risk model for problem (MV), respectively.

\section{Optimal Precommitted Investment Strategy for Problem (MV)}

This section will provide the precommitted investment strategy for problem (MV). We firstly state the main idea of solving problem (MV).

Let $d \in \mathbb{R}$ be fixed and consider the following problem with a constrained expectation:

$$
\left(\mathrm{MV}_{1}\right)\left\{\begin{array}{l}
E_{0, x}\left(X^{l}(T)\right)-\frac{\gamma}{2} \operatorname{Var}_{0, x}\left(X^{l}(T)\right) \longrightarrow \max \\
E_{0, x}\left(X_{T}^{l}\right)=d \\
l \in U .
\end{array}\right.
$$

Add the terminal condition $E_{0, x}\left(X_{T}^{l}\right)=d$ to the objective function and problem $\left(\mathrm{MV}_{1}\right)$ is equal to the following problem:

$$
\left(\mathrm{MV}_{2}\right)\left\{\begin{array}{l}
-\frac{\gamma}{2} E_{0, x}\left(X^{l}(T)\right)^{2}+d+\frac{\gamma}{2} d^{2} \longrightarrow \max \\
E_{0, x}\left(X_{T}^{l}\right)=d \\
l \in U
\end{array}\right.
$$

Denote the value function for problem $\left(\mathrm{MV}_{2}\right)$ by $V(x, d)$ and the value function $V(x)$ for problem (MV) satisfies $V(x)=$ $\sup _{d \in R} V(x, d)$. Problem $\left(\mathrm{MV}_{2}\right)$ can be solved by introducing a lagrange multiplier $y(x, d)$ and for $y:=y(x, d) \in \mathbb{R}$ define a quadratic utility problem

$$
\left(\mathrm{MV}_{3}\right)\left\{\begin{array}{c}
E_{0, x}\left[y X^{l}(T)-\frac{\gamma}{2}\left(X^{l}(T)\right)^{2}\right] \\
+d+\frac{\gamma}{2} d^{2}-d y \longrightarrow \max \\
l \in U .
\end{array}\right.
$$

Denote the value function for problem $\left(\mathrm{MV}_{3}\right)$ by $V(x, d, y)$. The duality theory implies that the value function for problem $\left(\mathrm{MV}_{2}\right)$ satisfies that

$$
V(x, d)=\inf _{y \in \mathbb{R}} V(x, d, y)
$$


The precommitted investment strategy for problem (MV) is derived by the following three steps.

Step 1. We will calculate the optimal investment strategy and the value function by solving the related Hamilton-JacobiBellman (HJB) equation for problem $\left(\mathrm{MV}_{3}\right)$. From standard arguments described as in Fleming and Soner [11], it is not hard to derive the following Verification Theorem on the HJB equation.

Lemma 2 (verification theorem). If there exist a real function $M(t, x) \in C^{1,2}(Q)$ and $l^{*} \in U$, which satisfy the following $H J B$ equation:

$$
\begin{gathered}
\sup _{l \in U}\left\{\mathscr{A}^{l} M(t, x)\right\}=0, \\
M(T, x)=-\frac{\gamma}{2} x^{2}+y x+\frac{\gamma}{2} d^{2}+d-y d, \\
l^{*}(t)=\arg \sup \{\mathscr{A} M(t, x)\},
\end{gathered}
$$

then $V(x, d, y)=M(0, x)$ and $l^{*}(t)$ is the optimal investment strategy.

Now, we will solve the HJB equation in Lemma 2. Assume that there exists a real function $M(t, x)$ which satisfies the boundary condition (15). By virtue of the infinitesimal operator (8), (14) can be rewritten as

$$
\begin{aligned}
\sup _{l \in U}\{ & M_{t}(t, x)+M_{x}(t, x)\left(r_{0}(t) x+c+r^{T}(t) l(t)\right) \\
+ & \frac{1}{2} M_{x x}(t, x)\left(l^{T}(t) \Sigma(t) l(t)+\sigma_{0}^{2}\right) \\
& +\lambda E[M(t, x+Z)-M(t, x)]\}=0 .
\end{aligned}
$$

Since both the structure of (17) and the boundary conditions of $M(t, x)$ are quadratic in $x$, we can conjecture that

$$
\begin{gathered}
M(t, x)=m(t) x^{2}+n(t) x+f(t), \\
m(T)=-\frac{\gamma}{2}, \quad n(T)=y, \quad f(T)=\frac{\gamma}{2} d^{2}+d-y d .
\end{gathered}
$$

Obviously,

$$
\begin{gathered}
M_{t}(t, x)=\dot{m}(t) x^{2}+\dot{n}(t) x+\dot{f}(t), \\
M_{x}(t, x)=2 m(t) x+n(t), \\
M_{x x}(t, x)=2 m(t) .
\end{gathered}
$$

Substituting (18)-(19) into (17), we have

$$
\begin{aligned}
\sup _{l \in U}\{ & \dot{m}(t) x^{2}+\dot{n}(t) x+\dot{f}(t)+(2 m(t) x+n(t)) \\
& \times\left(r_{0}(t) x+c+r^{T}(t) l(t)+\lambda \mu_{z}\right) \\
& \left.+m(t)\left(l^{T}(t) \Sigma(t) l(t)+\lambda \sigma_{z}^{2}+\sigma_{0}^{2}\right)\right\}=0 .
\end{aligned}
$$

Differentiating the function in the left bracket of (20) with respect to $l$ and setting the derivative to zero, we get

$$
(2 m(t) x+n(t)) r(t)+2 m(t) \Sigma(t) l(t)=0 .
$$

thus,

$$
l(t)=-\frac{(2 m(t) x+n(t)) \Sigma^{-1}(t) r(t)}{2 m(t)} .
$$

Inserting (22) into (20), we have

$$
\begin{aligned}
& \left(\dot{m}(t)+m(t)\left(2 r_{0}(t)-\xi(t)\right)\right) x^{2} \\
& \quad+\left(\dot{n}(t)+n(t)\left(r_{0}(t)-\xi(t)\right)+2 m(t) q\right) x \\
& \quad+\left(\dot{f}(t)+\left(\lambda \sigma_{z}^{2}+\sigma_{0}^{2}\right) m(t)+q n(t)-\frac{n^{2}(t)}{4 m(t)} \xi(t)\right)=0,
\end{aligned}
$$

where

$$
\xi(t)=r^{T}(t) \Sigma^{-1}(t) r(t) \geq 0, \quad q=\lambda \mu_{z}+c>0 .
$$

Letting the coefficients of $x$ and $x^{2}$ and the constant coefficient be equal to 0 in (23), we have

$$
\begin{gathered}
\dot{m}(t)+m(t)\left(2 r_{0}(t)-\xi(t)\right)=0, \quad m(T)=-\frac{\gamma}{2}, \\
\dot{n}(t)+n(t)\left(r_{0}(t)-\xi(t)\right)+2 m(t) q=0, \quad n(T)=y, \\
\dot{f}(t)+\left(\lambda \sigma_{z}^{2}+\sigma_{0}^{2}\right) m(t)+q n(t)-\frac{n^{2}(t)}{4 m(t)} \xi(t)=0, \\
f(T)=\frac{\gamma}{2} d^{2}+d-y d .
\end{gathered}
$$

The solutions of the ordinary differential equation (25) are as follows:

$$
\begin{gathered}
m(t)=-\frac{\gamma}{2} e^{\int_{t}^{T}\left(2 r_{0}(s)-\xi(s) d s\right.} \\
n(t)=e^{\int_{t}^{T}\left(r_{0}(s)-\xi(s) d s\right.}\left(-q \gamma \int_{t}^{T} e^{\int_{s}^{T} r_{0}(u) d u} d s+y\right) \\
f(t)=-\frac{\gamma\left(\lambda \sigma_{z}^{2}+\sigma_{0}^{2}\right)}{2} \int_{t}^{T} e^{\int_{s}^{T}\left(2 r_{0}(u)-\xi(u) d u\right.} d s \\
-\frac{1}{2 \gamma} e^{-\int_{t}^{T} \xi(u) d u}\left[-q \gamma \int_{t}^{T} e^{\int_{s}^{T} r_{0}(v) d v} d s+y\right]^{2} \\
+\frac{\gamma}{2} d^{2}+d-d y+\frac{y^{2}}{2 \gamma} .
\end{gathered}
$$

Substituting (26) and (27) into (22), we have

$$
\begin{aligned}
l(t)= & \Sigma^{-1}(t) r(t) \\
& \times\left\{e^{-\int_{t}^{T} r_{0}(s) d s}\left(-q \int_{t}^{T} e^{\int_{s}^{T} r_{0}(u) d u} d s+\frac{y}{\gamma}\right)-x(t)\right\} .
\end{aligned}
$$


According to the argument above, optimal investment strategy and the value function for problem $\left(\mathrm{MV}_{3}\right)$ are given by the following theorem.

Theorem 3. For problem $\left(M V_{3}\right)$, optimal investment strategy $l_{1}^{*}$ is given by

$$
\begin{aligned}
l_{1}^{*}(t)= & \Sigma^{-1}(t) r(t) \\
& \times\left\{e^{-\int_{t}^{T} r_{0}(s) d s}\left(-q \int_{t}^{T} e^{\int_{s}^{T} r_{0}(u) d u} d s+\frac{y}{\gamma}\right)-x(t)\right\}
\end{aligned}
$$

and the value function is given by

$$
\begin{aligned}
V(x, d, y) \\
=-\frac{\gamma\left(\lambda \sigma_{z}^{2}+\sigma_{0}^{2}\right)}{2} \int_{0}^{T} e^{\int_{s}^{T}\left(2 r_{0}(u)-\xi(u) d u\right.} d s \\
\quad-\frac{\gamma}{2} e^{-\int_{0}^{T} \xi(s) d s}\left\{x e^{\int_{0}^{T} r_{0}(s) d s}+\left[q \int_{0}^{T} e^{\int_{s}^{T} r_{0}(u) d u} d s-\frac{y}{\gamma}\right]\right\}^{2} \\
\quad+\frac{\gamma}{2} d^{2}+d-d y+\frac{y^{2}}{2 \gamma} .
\end{aligned}
$$

Step 2. By virtue of $V(x, d)=\inf _{y \in \mathbb{R}} V(x, d, y)$, we can solve problem $\left(\mathrm{MV}_{2}\right)$. Differentiating $V(x, d, y)$ with respect to $y$, we have

$$
\begin{aligned}
\frac{\partial V}{\partial y}= & e^{-\int_{0}^{T} \xi(s) d s}\left\{x e^{\int_{0}^{T} r_{0}(s) d s}+\left[q \int_{0}^{T} e^{\int_{s}^{T} r_{0}(u) d u} d s-\frac{y}{\gamma}\right]\right\} \\
& -d+\frac{y}{\gamma}
\end{aligned}
$$

Setting the derivative to zero yields that

$$
y^{*}=\frac{\left[q \int_{0}^{T} e^{\int_{s}^{T} r_{0}(v) d v} d s+x e^{\int_{0}^{T} r_{0}(s) d s}-d e^{\int_{0}^{T} \xi(s) d s}\right] \gamma}{1-e^{\int_{0}^{T} \xi(s) d s}} .
$$

Furthermore,

$$
\frac{\partial^{2} V}{\partial y^{2}}=\frac{1-e^{-\int_{0}^{T} \xi(s) d s}}{\gamma}>0 .
$$

Therefore $y^{*} \in \mathbb{R}$ is the point which minimizes $V(x, d, y)$ according to the extreme value theory. By inserting (33) into (30)-(31), optimal investment strategy $l_{2}^{*}(t)$ and the value function $V(x, d)$ for problem $\left(\mathrm{MV}_{2}\right)$ are given by the following theorem.
Theorem 4. For problem $\left(\mathrm{MV}_{2}\right)$, optimal investment strategy $l_{2}^{*}$ is given by

$$
\begin{aligned}
& l_{2}^{*}(t) \\
& =\Sigma^{-1}(t) r(t) \\
& \times \quad\left\{e^{-\int_{t}^{T} r_{0}(s) d s}\right. \\
& \quad \times\left(-q \int_{t}^{T} e^{\int_{s}^{T} r_{0}(u) d u} d s\right. \\
& \left.\quad+\frac{q \int_{0}^{T} e^{\int_{s}^{T} r_{0}(v) d v} d s+x e^{T_{0}^{T} r_{0}(s) d s}-d e^{\int_{0}^{T} \xi(s) d s}}{1-e \int_{0}^{T} \xi(s) d s}\right) \\
& \quad-x(t)\}
\end{aligned}
$$

and the value function is given by

$$
\begin{aligned}
V(x, d)= & -\frac{\gamma \lambda \sigma_{z}^{2}}{2} \int_{0}^{T} e^{\int_{s}^{T}\left(2 r_{0}(u)-\xi(u) d u\right.} d s \\
& +\frac{\gamma}{2\left(1-e^{\int_{0}^{T} \xi(s) d s}\right)} \\
& \times\left\{x e^{\int_{0}^{T} r_{0}(s) d s}+q \int_{0}^{T} e^{\int_{s}^{T} r_{0}(u) d u} d s-d\right\}^{2}+d .
\end{aligned}
$$

Step 3. Problem (MV) can be finally solved by virtue of the relationship of $V(x)$ and $V(x, d)$. Differentiating $V(x, d)$ at $d$ yields that

$$
\begin{aligned}
\frac{\partial V(x, d)}{\partial d}= & \frac{\gamma}{1-e^{\int_{0}^{T} \xi(s) d s}} \\
& \times\left\{d-x e^{\int_{0}^{T} r_{0}(s) d s}-q \int_{0}^{T} e^{\int_{s}^{T} r_{0}(u) d u} d s\right\}+1,
\end{aligned}
$$

$$
\frac{\partial^{2} V(x, d)}{\partial d^{2}}=\frac{\gamma}{1-e^{\int_{0}^{T} \xi(s) d s}}<0
$$

From the extreme value theory, the optimal expected terminal wealth $d^{*}$ does exist and satisfies $\partial V(x, d) / \partial d=0$. By a simple calculation, we have

$$
d^{*}=q \int_{0}^{T} e^{\int_{s}^{T} r_{0}(v) d v} d s+x e^{\int_{0}^{T} r_{0}(s) d s}+\frac{\left(e^{\int_{0}^{T} \xi(s) d s}-1\right)}{\gamma} .
$$

Therefore, inserting (38) into (35) and (36), optimal investment strategy and the value function for problem (MV) can be derived explicitly and they are given by the following theorem. 
Theorem 5. For problem (MV), optimal precommitted investment strategy $l^{*}$ is given by

$l^{*}(t)$

$$
\begin{aligned}
& =\Sigma^{-1}(t) r(t) \\
& \times\left\{e^{-\int_{t}^{T} r_{0}(s) d s}\right. \\
& \quad \times\left(q \int_{0}^{t} e^{\int_{s}^{T} r_{0}(v) d v} d s+x e^{\int_{0}^{T} r_{0}(s) d s}+\frac{e^{\int_{0}^{T} \xi(s) d s}}{\gamma}\right) \\
& \quad-x(t)\}
\end{aligned}
$$

and the value function is given by

$$
\begin{aligned}
& V(x)=x e^{\int_{0}^{T} r_{0}(s) d s}+q \int_{0}^{T} e^{\int_{s}^{T} r_{0}(u) d u} d s \\
& -\frac{\gamma\left(\lambda \sigma_{z}^{2}+\sigma_{0}^{2}\right)}{2} \int_{0}^{T} e^{\int_{s}^{T}\left(2 r_{0}(u)-\xi(u) d u\right.} d s \\
& +\frac{\left(e^{\int_{0}^{T} \xi(s) d s}-1\right)}{2 \gamma} \text {. }
\end{aligned}
$$

Remark 6. The efficient frontier at initial state $(0, x)$ can be derived. By Theorem 5 and the definition of $d^{*}$, we have

$$
\begin{aligned}
E_{0, x}\left(X^{l^{*}}(T)\right)= & q \int_{0}^{T} e^{\int_{s}^{T} r_{0}(v) d v} d s+x e^{\int_{0}^{T} r_{0}(s) d s} \\
& +\frac{\left(e^{\int_{0}^{T} \xi(s) d s}-1\right)}{\gamma}, \\
\operatorname{Var}_{0, x}\left(X^{l^{*}}(T)\right)= & \left(\lambda \sigma_{z}^{2}+\sigma_{0}^{2}\right) \int_{0}^{T} e^{\int_{s}^{T}\left(2 r_{0}(u)-\xi(u) d u\right.} d s \\
& +\frac{1}{\gamma^{2}}\left(e^{\int_{0}^{T} \xi(s) d s}-1\right) .
\end{aligned}
$$

So the efficient frontier at initial state $(0, x)$ is as follows:

$$
\begin{aligned}
E_{0, x}\left(X^{l^{*}}(T)\right)=q & \int_{0}^{T} e^{\int_{s}^{T} r_{0}(v) d v} d s+x e^{\int_{0}^{T} r_{0}(s) d s} \\
+ & \left(\left[\operatorname{Var}_{0, x}\left(X^{l^{*}}(T)\right)\right.\right. \\
& \left.-\left(\lambda \sigma_{z}^{2}+\sigma_{0}^{2}\right) \int_{0}^{T} e^{\int_{s}^{T}\left(2 r_{0}(u)-\xi(u) d u\right.} d s\right] \\
& \left.\times\left(e^{\int_{0}^{T} \xi(s) d s}-1\right)\right)^{1 / 2} .
\end{aligned}
$$

This efficient frontier is not a straight line but a hyperbola in the mean-standard deviation plane.
Remark 7. The precommitted investment strategy is stochastically dependent on the current wealth which means that $l^{*}(t)$ is a stochastic process which satisfies the following stochastic differential equation:

$$
\begin{aligned}
d X^{l^{*}}(t)= & \left(r_{0}(t) X^{l^{*}}(t)+r^{T}(t) l^{*}(t)+c\right) d t \\
& +\left(l^{*}(t)\right)^{T} \sigma(t) d W(t)+d \sum_{j=1}^{N(t)} Z_{j}+\sigma_{0} W_{0}(t) .
\end{aligned}
$$

So all the parameters impact the precommitted investment strategy together, and we can only analyze the effect of the parameters on $l^{*}(t)$ by numerical simulation.

Remark 8 . When all the parameters are all constants and $d=n=1$, the optimal precommitted investment strategy, the corresponding value function, and the efficient frontier are given by the following equations:

$$
\begin{aligned}
l^{*}(t)=\frac{r_{1}-r_{0}}{\sigma^{2}}\left\{\frac{\lambda \mu_{z}+c}{r_{0}}\left(e^{r_{0} t}-1\right)+x e^{r_{0} t}\right. & \\
& \left.+\frac{e^{\left[\left(r_{1}-r_{0}\right)^{2} / \sigma^{2}-r_{0}\right] T} e^{r_{0} t}}{\gamma}-x(t)\right\},
\end{aligned}
$$

$$
\begin{aligned}
V(x)= & x e^{r_{0} T}+\frac{\lambda \mu_{z}+c}{r_{0}}\left(e^{r_{0} T}-1\right) \\
& -\frac{\gamma\left(\lambda \sigma_{z}^{2}+\sigma_{0}^{2}\right)}{2\left(2 r_{0}-\left(r_{1}-r_{0}\right)^{2} / \sigma^{2}\right)}\left(e^{\left(2 r_{0}-\left(r_{1}-r_{0}\right)^{2} / \sigma^{2}\right) T}-1\right) \\
& +\frac{\left(e^{\left(\left(r_{1}-r_{0}\right)^{2} / \sigma^{2}\right) T}-1\right)}{2 \gamma},
\end{aligned}
$$

$$
\begin{aligned}
& E_{0, x}\left(X^{l^{*}}(T)\right) \\
& =\left(\left[\operatorname{Var}_{0, x}\left(X^{l^{*}}(T)\right)-\frac{\left(\lambda \sigma_{z}^{2}+\sigma_{0}^{2}\right)}{\left(2 r_{0}-\left(r_{1}-r_{0}\right)^{2} / \sigma^{2}\right)}\right.\right. \\
& \left.\quad \times\left(e^{\left(2 r_{0}-\left(r_{1}-r_{0}\right)^{2} / \sigma^{2}\right) T}-1\right)\right] \\
& \left.\quad \times\left(e^{\left(\left(r_{1}-r_{0}\right)^{2} / \sigma^{2}\right) T}-1\right)\right)^{1 / 2}
\end{aligned}
$$$$
+x e^{r_{0} T}+\frac{\lambda \mu_{z}+c}{r_{0}}\left(e^{r_{0} T}-1\right)
$$ 


\section{Optimal Time-Consistent Investment Strategy for Problem (MV)}

In this section, we will provide optimal time-consistent investment strategy and the equilibrium value function for problem (MV) by solving the extended HJB equations.

Firstly define problem $(\overline{\mathrm{MV}})$

$$
(\overline{\mathrm{MV}})\left\{\begin{array}{l}
E_{t, x}\left[X^{l}(T)\right]-\frac{\gamma}{2} \operatorname{Var}_{t, x}\left[X^{l}(T)\right] \longrightarrow \max \\
l \in U,
\end{array}\right.
$$

and denote the value function by $\psi(t, x)$ for problem $(\overline{\mathrm{MV}})$. Due to the fact that this objective function is nonlinear in the expectation of the terminal surplus, problem $(\overline{\mathrm{MV}})$ is time-inconsistent in the sense that the Bellman Optimality Principle does not hold. In order to deal with this timeinconsistent problem, we can view the investment problem as a noncooperative game with one player for each time $t$ and look for some equilibrium strategy which will also be equilibrium for any time $t \in[0, T]$. The definitions of equilibrium strategy and verification theorem for problem $(\overline{\mathrm{MV}})$ are described as similarly as in Björk and Murgoci [4] or Zeng et al. [10].

Definition 9 (equilibrium strategy). For any fixed chosen initial state $(t, x) \in Q$, consider an admissible strategy $l_{*}(t, x)$. Choose two fixed real numbers $\tilde{l}>0$ and $\varepsilon>0$ and define the following strategy:

$$
l^{\varepsilon}(s, x)= \begin{cases}\widetilde{l}, & \text { for }(s, x) \in[t, t+\varepsilon] \times \mathbb{R} \\ l_{*}(s, x), & \text { for }(s, x) \in[t+\varepsilon, T] \times \mathbb{R} .\end{cases}
$$

If

$$
\liminf _{\varepsilon \rightarrow 0} \frac{J\left(t, x, l_{*}\right)-J\left(t, x, l^{\varepsilon}\right)}{\varepsilon} \geq 0, \quad \forall \widetilde{l} \in \mathbb{R}^{+},(t, x) \in Q,
$$

then $l_{*}(t, x)$ is called an equilibrium strategy, and the corresponding equilibrium value function is defined by

$$
\psi(t, x)=J\left(t, x, l_{*}\right)=E_{t, x}\left[X^{l_{*}}(T)\right]-\frac{\gamma}{2} \operatorname{Var}_{t, x}\left[X^{l_{*}}(T)\right] .
$$

It is easy to see that the equilibrium strategy is timeconsistent. So the equilibrium strategy $l_{*}$ is called optimal time-consistent strategy.

Lemma 10 (verification theorem). If there exist two real functions $Q(t, x)$ and $g(t, x) \in C^{1,2}(Q)$, satisfying the following extended HJB equations:

$$
\begin{gathered}
\sup _{l \in U}\left\{\mathscr{A}^{l} Q(t, x)-\mathscr{A}^{l}\left(\frac{\gamma}{2} g^{2}(t, x)\right)+\gamma g(t, x) \mathscr{A}^{l} g(t, x)\right\}=0 \\
Q(T, x)=x \\
\mathscr{A}^{l_{*}} g(t, x)=0 \\
g(T, x)=x
\end{gathered}
$$

where

$$
\begin{aligned}
l_{*}=\arg \sup \{ & \mathscr{A} Q(t, x)-\mathscr{A}\left(\frac{\gamma}{2} g^{2}(t, x)\right) \\
& +\gamma g(t, x) \mathscr{A} g(t, x)\},
\end{aligned}
$$

then $\psi(t, x)=Q(t, x), E_{t, x}\left(X^{l_{*}}(t)\right)=g(t, x)$, and $l_{*}$ is optimal time-consistent strategy.

Next, we will find the solution to the extended HJB equations. By using the infinitesimal generator (8), we can rewrite the extended HJB equations in Lemma 10 as

$$
\begin{aligned}
& \sup _{l \in U}\left\{Q_{t}(t, x)+Q_{x}(t, x)\left(r_{0}(t) x+c+r^{T}(t) l(t)\right)\right. \\
& +\frac{1}{2}\left(Q_{x x}(t, x)-\gamma g_{x}^{2}(t, x)\right)\left(l^{T}(t) \Sigma(t) l(t)+\sigma_{0}^{2}\right) \\
& +\lambda E\left[Q(t, x+Z)-\frac{\gamma}{2} g(t, x+Z)\right. \\
& \quad \times(g(t, x+Z)-2 g(t, x))] \\
& \left.-\lambda\left[Q(t, x)+\frac{\gamma}{2} g^{2}(t, x)\right]\right\}=0, \\
& g_{t}(t, x)+g_{x}(t, x)\left(r_{0}(t) x+c+r^{T}(t) l_{*}(t)\right) \\
& +\frac{1}{2} g_{x x}(t, x)\left(l_{*}^{T}(t) \Sigma(t) l_{*}(t)+\sigma_{0}^{2}\right) \\
& +\lambda E[g(t, x+Z)-g(t, x)]=0,
\end{aligned}
$$

where $l_{*}(t)$ is determined below.

On one hand, differentiating the function in the left bracket of (56) with respect to $l$ and setting the derivative to zero, we get

$$
l_{*}(t)=-\frac{\Sigma^{-1}(t) r(t) Q_{x}(t, x)}{Q_{x x}(t, x)-\gamma g_{x}^{2}(t, x)} .
$$

On the other hand, since the linear structure of (56) and (57) and the boundary conditions of $Q(t, x)$ and $g(t, x)$ given by (52) and (54) are linear in $x$, we can guess that

$$
\begin{array}{cl}
Q(t, x)=A(t) x+D(t), & A(T)=1, D(T)=0, \\
g(t, x)=a(t) x+d(t), & a(T)=1, d(T)=0 .
\end{array}
$$

Thus, the partial derivatives for the functions $Q(t, x)$ and $g(t$, $x$ ) are easily calculated:

$$
\begin{array}{r}
Q_{t}(t, x)=\dot{A}(t) x+\dot{D}(t), \\
Q_{x}(t, x)=A(t), \quad Q_{x x}(t, x)=0, \\
g_{t}(t, x)=\dot{a}(t) x+\dot{d}(t), \\
g_{x}(t, x)=a(t), \quad g_{x x}(t, x)=0 .
\end{array}
$$


Substituting (60) into (58) yields

$$
l_{*}(t)=\frac{A(t) \Sigma^{-1}(t) r(t)}{\gamma a^{2}(t)} .
$$

Inserting (59)-(61) into (56)-(57), we have

$$
\begin{aligned}
& \left(\dot{A}(t)+r_{0}(t) A(t)\right) x+\dot{D}(t)+c A(t)+\lambda \mu_{z} A(t) \\
& -\frac{\gamma}{2} a^{2}(t)\left(\lambda \sigma_{z}^{2}+\sigma_{0}^{2}\right)+\frac{A^{2}(t) \xi(t)}{2 \gamma a^{2}(t)}=0, \\
& \left(\dot{a}(t)+r_{0}(t) a(t)\right) x+\dot{d}(t)+c a(t)+\lambda \mu_{z} a(t) \\
& \quad+\frac{A(t) \xi(t)}{\gamma a(t)}=0,
\end{aligned}
$$

where

$$
\xi(t)=r^{T}(t) \Sigma^{-1}(t) r(t) \geq 0
$$

Because (62) holds for $\forall x \in \mathbb{R}$ which means that the coefficient of $x$ and the constant coefficient are equal to 0 , we have

$$
\begin{gathered}
\dot{A}(t)+r_{0}(t) A(t)=0, \quad A(T)=1, \\
\dot{D}(t)+\left(\lambda \mu_{z}+c\right) A(t)-\frac{\gamma}{2} a^{2}(t)\left(\lambda \sigma_{z}^{2}+\sigma_{0}^{2}\right) \\
+\frac{A^{2}(t) \xi(t)}{2 \gamma a^{2}(t)}=0, \quad D(T)=0, \\
\dot{a}(t)+r_{0}(t) a(t)=0, \quad a(T)=1, \\
\dot{d}(t)+\left(\lambda \mu_{z}+c\right) a(t)+\frac{A(t) \xi(t)}{\gamma a(t)}=0, \quad d(T)=0 .
\end{gathered}
$$

The solutions of the system to ordinary equations are given as follows:

$$
\begin{gathered}
A(t)=e^{\int_{t}^{T} r_{0}(s) d s} \\
D(t)=\left(\lambda \mu_{z}+c\right) \int_{t}^{T} e^{\int_{s}^{T} r_{0}(u) d u} d s \\
-\frac{\gamma}{2}\left(\lambda \sigma_{z}^{2}+\sigma_{0}^{2}\right) \int_{t}^{T} e^{2 \int_{s}^{T} r_{0}(u) d u} d s \\
+\frac{1}{2 \gamma} \int_{t}^{T} \xi(s) d s, \\
a(t)=e^{\int_{t}^{T} r_{0}(s) d s}, \\
d(t)=\left(\lambda \mu_{z}+c\right) \int_{t}^{T} e^{\int_{s}^{T} r_{0}(u) d u} d s+\frac{1}{\gamma} \int_{t}^{T} \xi(s) d s .
\end{gathered}
$$

By inserting (65) and (67) into (61), the optimal timeconsistent strategy is given by the following equation:

$$
l_{*}(t)=\frac{\Sigma^{-1}(t) r(t)}{\gamma} e^{-\int_{t}^{T} r_{0}(s) d s} .
$$

Based on the argument above, the explicit expressions for $Q(t, x)$ and $g(t, x)$ are obtained. Let the original time $t$ for problem $(\overline{\mathrm{MV}})$ equal 0 and the value function for problem (MV) is given by the following theorem.

Theorem 11. For problem (MV), optimal time-consistent strategy $l_{*}$ is given by (69) and the equilibrium value function is given by the following equation:

$$
\begin{aligned}
V^{*}(x)= & e^{\int_{0}^{T} r_{0}(s) d s} x+\left(\lambda \mu_{z}+c\right) \int_{0}^{T} e^{\int_{s}^{T}} r_{0}(u) d u d s \\
& -\frac{\gamma\left(\lambda \sigma_{z}^{2}+\sigma_{0}^{2}\right)}{2} \int_{0}^{T} e^{2 \int_{s}^{T} r_{0}(u) d u} d s \\
& +\frac{1}{2 \gamma} \int_{0}^{T} \xi(s) d s .
\end{aligned}
$$

Furthermore,

$$
\begin{aligned}
E_{0, x}\left(X^{l_{*}}(T)\right)=g(0, x)= & e^{\int_{0}^{T} r_{0}(s) d s} x \\
& +\left(\lambda \mu_{z}+c\right) \int_{0}^{T} e^{\int_{s}^{T} r_{0}(u) d u} d s \\
& +\frac{1}{\gamma} \int_{0}^{T} \xi(s) d s .
\end{aligned}
$$

Remark 12. By virtue of (70) and (71), the relationship between the expectation and the variance of the terminal wealth is derived:

$$
\begin{aligned}
E_{0, x}\left(X^{l_{*}}(T)\right)= & e^{\int_{0}^{T} r_{0}(s) d s} x+\left(\lambda \mu_{z}+c\right) \int_{0}^{T} e^{\int_{s}^{T} r_{0}(u) d u} d s \\
+ & \left(\left[\operatorname{Var}_{0, x}\left(X^{l_{*}}(T)\right)-\left(\lambda \sigma_{z}^{2}+\sigma_{0}^{2}\right)\right.\right. \\
& \left.\left.\times \int_{0}^{T} e^{2 \int_{s}^{T} r_{0}(u) d u} d s\right] \int_{0}^{T} \xi(s) d s\right)^{1 / 2} .
\end{aligned}
$$

Equation (72) also shows this efficient frontier is a hyperbola in the mean-standard deviation plane.

Remark 13. This time-consistent investment strategy is independent on the current wealth which means $l_{*}(t)$ is a deterministic function with respect to $t$. The parameters of the surplus process have no impact on the optimal strategy; the risk aversion coefficient and the coefficients of financial market decide the optimal strategy together. 

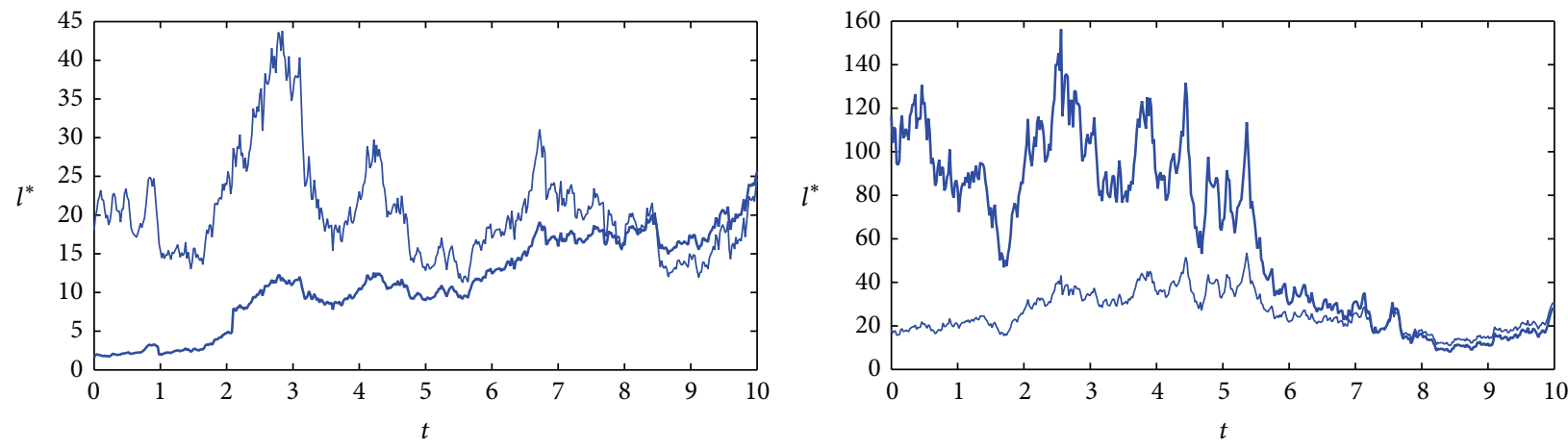

$-r_{0}=0.03$

$-r_{1}=0.12$

(a) The effect of $r_{0}$ on optimal precommitted strategy

(b) The effect of $r_{1}$ on optimal precommitted strategy
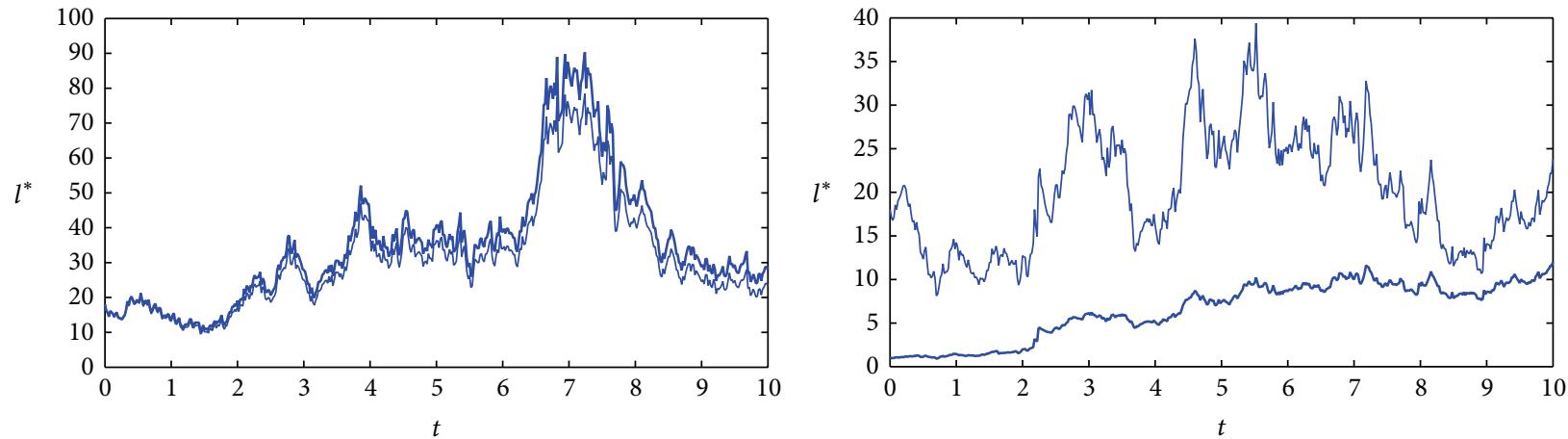

$-\lambda=1$

$-\sigma=0.2$

$\sigma=0.4$

(c) The effect of $\lambda$ on optimal precommitted strategy

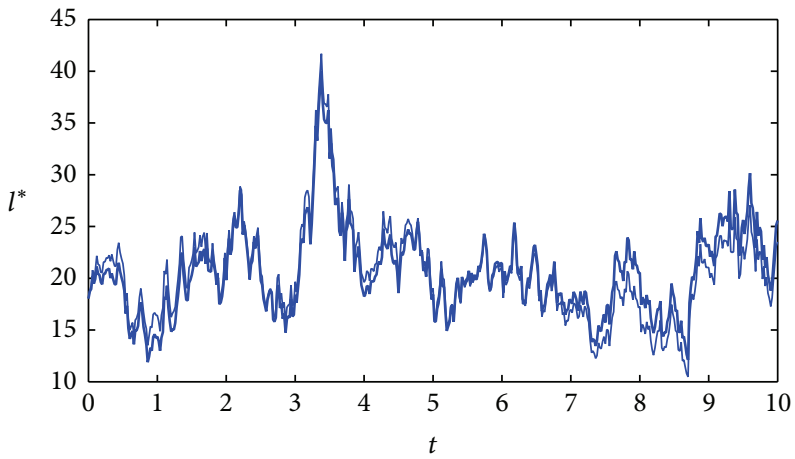

(d) The effect of $\sigma$ on optimal precommitted strategy

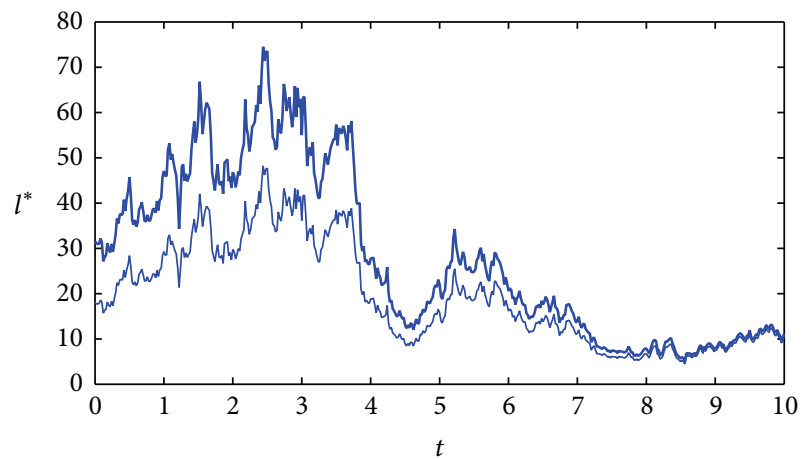

$\sigma_{0}=0.2$

$-\gamma=0.7$

$-\gamma=0.4$

(e) The effect of $\sigma_{0}$ on optimal precommitted strategy

(f) The effect of $\gamma$ on optimal precommitted strategy

FIGURE 1: The effect of parameters on optimal precommitted strategy for the general risk model.

Remark 14. When all the parameters are all constants and $d=$ $n=1$, optimal time-consistent strategy $l_{*}$, the equilibrium value function $V^{*}(x)$, and the efficient frontier are given by

$$
l_{*}(t)=\frac{r_{1}-r_{0}}{\gamma \sigma^{2}} e^{-r_{0}(T-t)},
$$

$$
\begin{aligned}
V^{*}(x)= & x e^{r_{0} T}+\frac{\lambda \mu_{z}+c}{r_{0}}\left(e^{r_{0} T}-1\right) \\
& -\frac{\gamma\left(\lambda \sigma_{z}^{2}+\sigma_{0}^{2}\right)}{4 r_{0}}\left(e^{2 r_{0} T}-1\right)+\frac{1}{2 \gamma} \frac{\left(r_{1}-r_{0}\right)^{2}}{\sigma^{2}} T,
\end{aligned}
$$




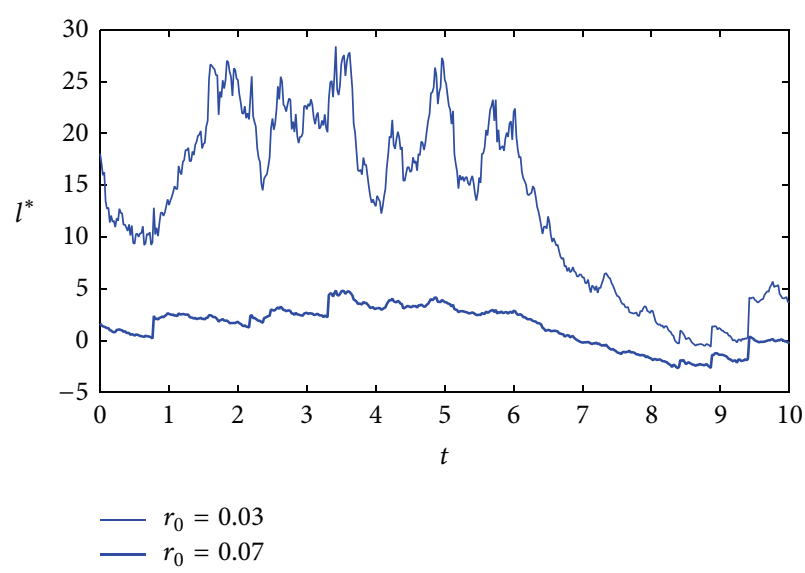

(a) The effect of $r_{0}$ on optimal precommitted strategy

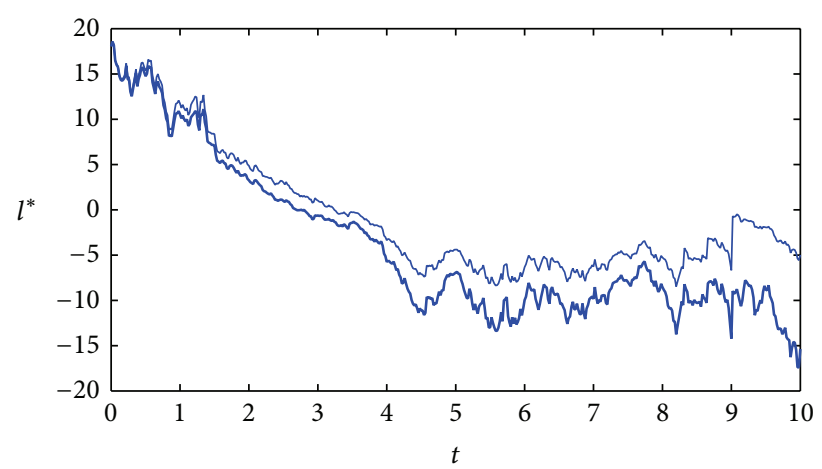

$-\lambda=1$

(c) The effect of $\lambda$ on optimal precommitted strategy

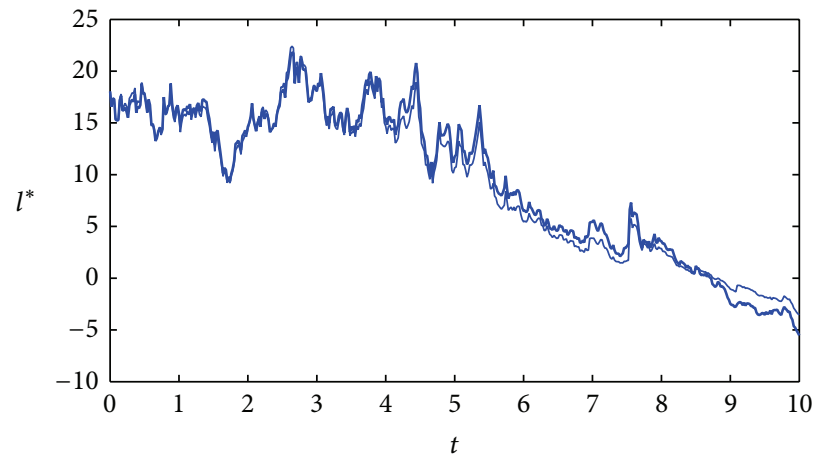

$-\sigma_{0}=0.2$

- $\sigma_{0}=0.6$

(e) The effect of $\sigma_{0}$ on optimal precommitted strategy

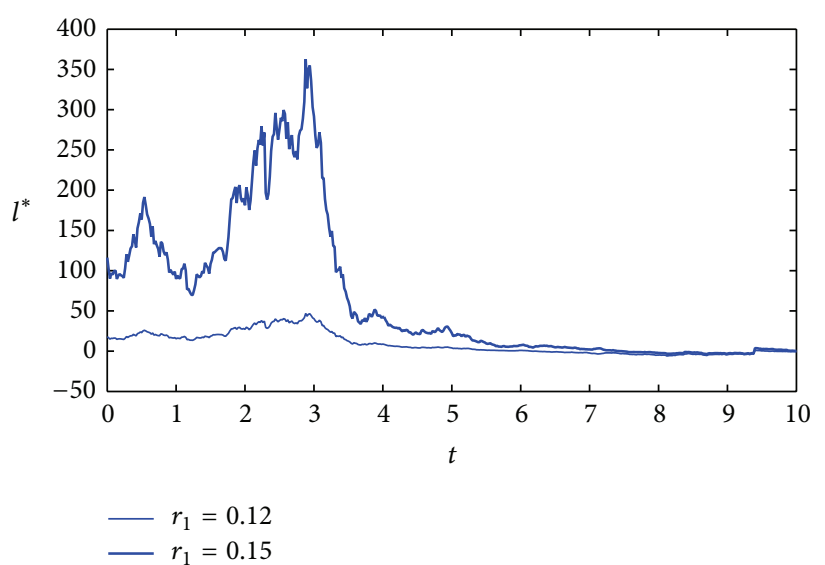

(b) The effect of $r_{1}$ on optimal precommitted strategy

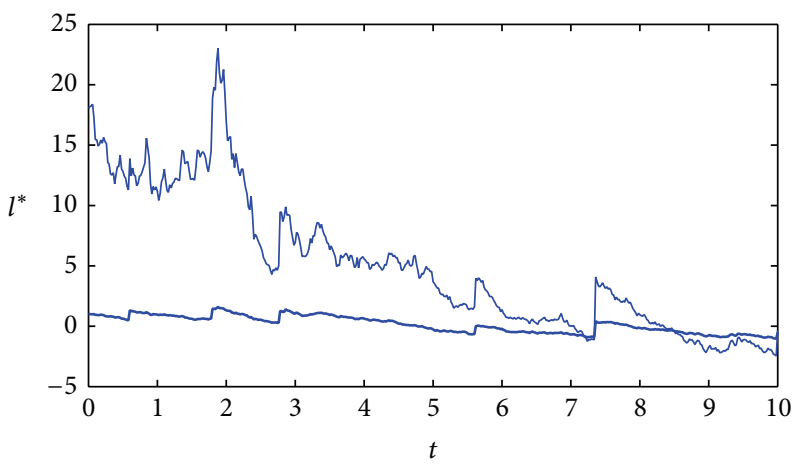

$-\sigma=0.2$

$\sigma=0$.

(d) The effect of $\sigma$ on optimal precommitted strategy

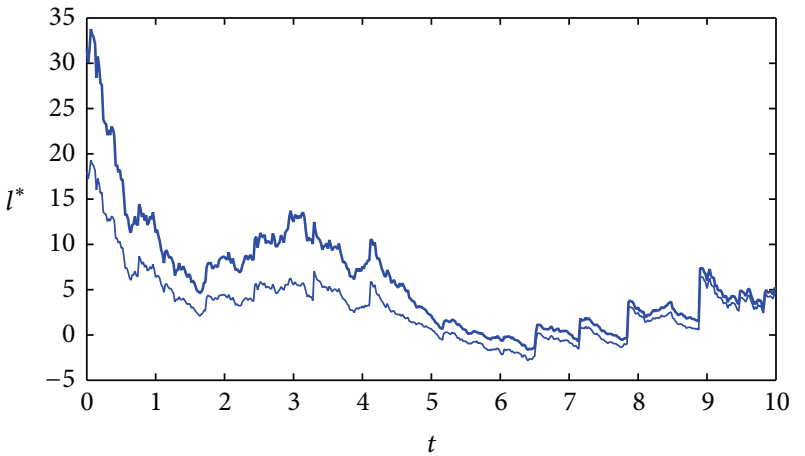

$-\gamma=0.7$

$-\gamma=0.4$

(f) The effect of $\gamma$ on optimal precommitted strategy

FIGURE 2: The effect of parameters on optimal precommitted strategy for the C-L risk model.

$$
\begin{aligned}
E_{0, x}\left(X^{l_{*}}(T)\right) & \\
= & x e^{r_{0} T}+\frac{\lambda \mu_{z}+c}{r_{0}}\left(e^{r_{0} T}-1\right) \\
& +\left(\left[\operatorname{Var}_{0, x}\left(X^{l_{*}}(T)\right)-\frac{\lambda \sigma_{z}^{2}+\sigma_{0}^{2}}{2 r_{0}}\left(e^{2 r_{0} T}-1\right)\right]\right. \\
& \left.\times \frac{\left(r_{1}-r_{0}\right)^{2}}{\sigma^{2}} T\right)^{1 / 2} .
\end{aligned}
$$

\section{Numerical Analysis}

In the next two subsections, we study the effect of parameters on the optimal strategies (precommitted strategy and timeconsistent strategy) and the corresponding value functions and provide some numerical examples to illustrate the effects. Finally, compare the precommitment results with the timeconsistent ones by some numerical analysis. For convenience but without loss of generality, all the parameters involved are 


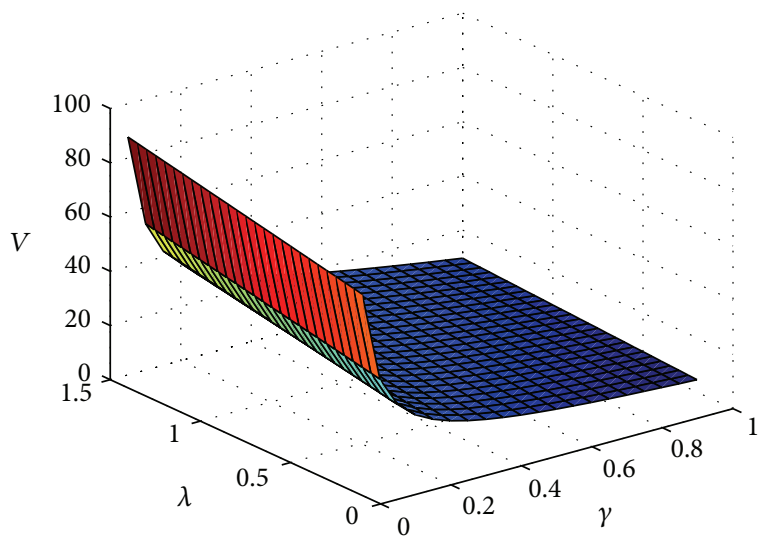

(a) The effect of $\gamma$ and $\lambda$ on the value function

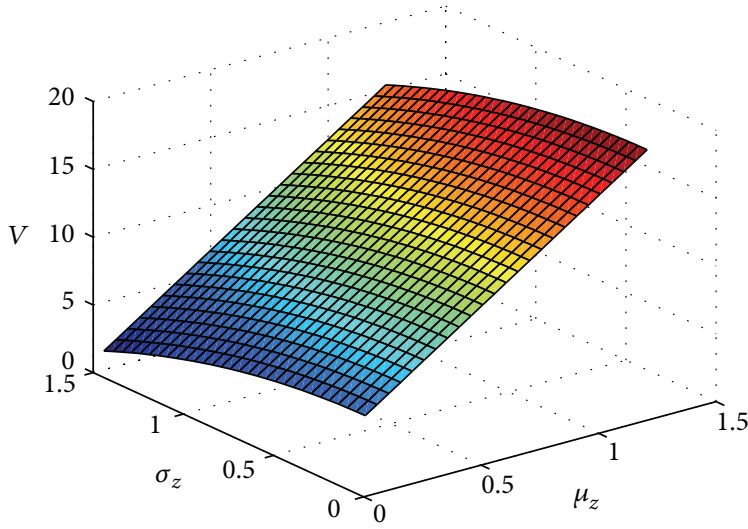

(c) The effect of $\mu_{z}$ and $\sigma_{z}$ on the value function

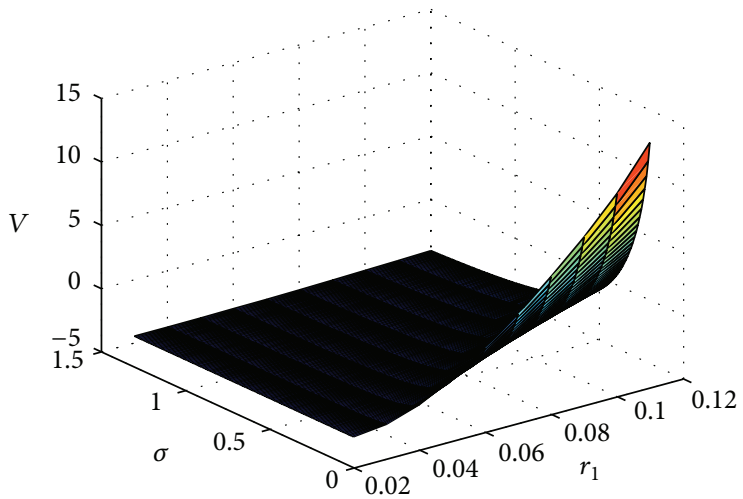

(b) The effect of $r_{1}$ and $\sigma$ on the value function

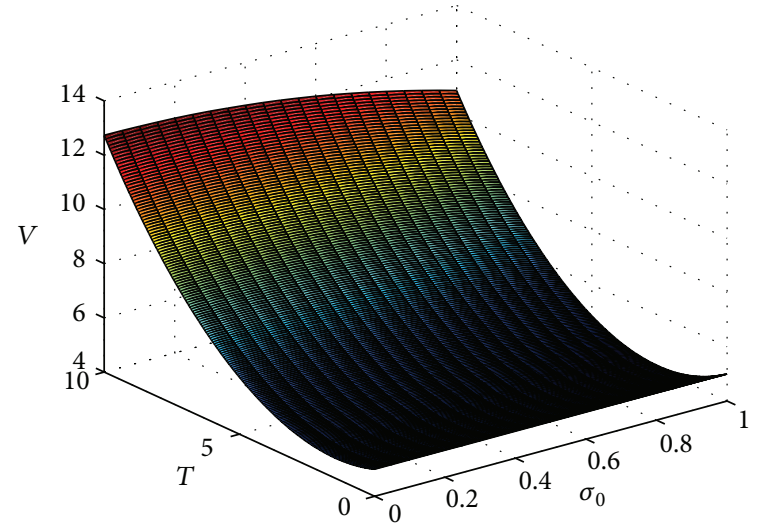

(d) The effect of $\sigma_{0}$ and $T$ on the value function

FIgURE 3: The effect of parameters on the value function.

constants and $d=n=1$. The optimal investment strategies, the corresponding value functions, and the efficient frontiers are given by Remarks 8 and 14 from different views. For the following numerical illustrates, unless otherwise stated, the basic parameters are given by $r_{0}=0.03, r_{1}=0.12, c=-0.5$, $x=5, \gamma=0.7, \lambda=1, \mu_{z}=1.25, \sigma_{z}=2.0, \sigma=0.2, \sigma_{0}=0.4$, and $T=10$.

5.1. Analysis of Optimal Precommitted Strategy and the Corresponding Value Function. In this subsection, we will work on numerical analysis of the precommitted strategy and the value function.

Firstly, we will show how the coefficients involved impact on the precommitted strategy. Since the precommitted investment strategy is stochastically dependent on the current wealth, we explore the effect of parameters of the financial market and the risk aversion by stochastic simulation. Because the precommitted strategy is indeed a stochastic process, we investigate the effect of different parameters in a same sample trajectory. In order to model the trajectory, we assume that $\{N(t)\}_{t \geq 0}$ is a Poisson process with intensity $\lambda$ and the profits or the incomes $Z_{j}(j=1,2,3 \ldots)$ are double exponentially distributed with parameters $p, \alpha$, and $\beta$; namely, its probability density function is given by $f_{z}(z)=$ $p \times \alpha e^{-\alpha z} I_{z>0}+(1-p) \times \beta e^{\beta z} I_{z<0}$. From (44), we can see that the optimal precommitted investment strategy increases when the current wealth decreases; namely, if the current wealth is big enough, the company should invest less money in the risk-risky asset. In order to simulate the general risk model assume $p=0.4, \alpha=0.2$, and $\beta=0.8$ and Figure 1 shows how the coefficients involved impact the optimal precommitted investment strategy for the general risk model with diffusion. In order to simulate the C-L risk model, assume $p=0$, $\alpha=0.2, \beta=0.8$, and $c=1.5$ and Figure 2 shows how the coefficients involved impact the optimal precommitted investment strategy for the C-L risk model with diffusion. From Figures 1 and 2, we can conclude the following findings: all the parameters impact the precommitted strategy together and optimal precommitted investment strategy has more complex relation with all the parameters, because the increase of one parameter can change the deterministic part of the precommitted strategy and the current wealth together which results in the uncertainty of their difference.

Secondly, we will show how the coefficients involved impact the value function. For convenience, introduce the notation $a=\left(2 r_{0}-\left(r_{1}-r_{0}\right)^{2} / \sigma^{2}\right) T$ and we can show that $\left(e^{a}-1\right) / a>0$ and $e^{a} a-\left(e^{a}-1\right) \geq 0$ for all $a \in \mathbb{R}$ by an elementary calculation. From (45), we can conclude the following findings. 


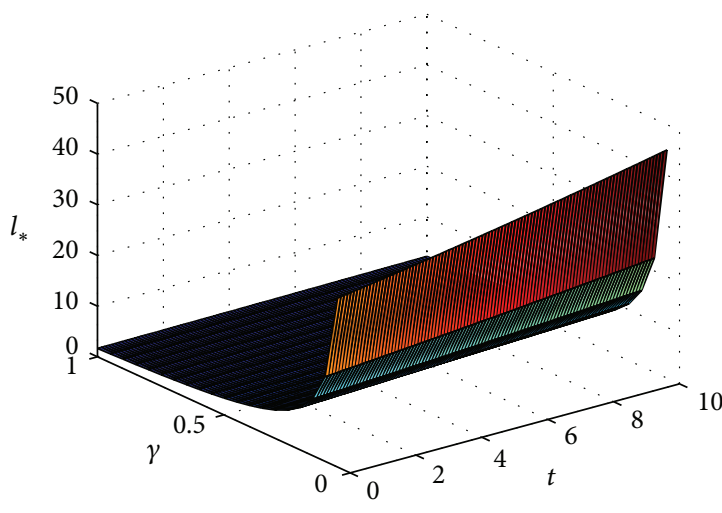

(a) The effect of $\gamma$ and $t$ on optimal time-consistent strategy

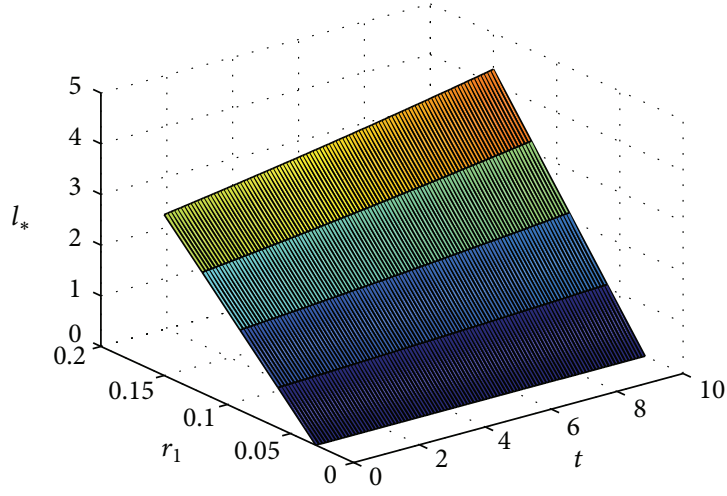

(c) The effect of $r_{1}$ and $t$ on optimal time-consistent strategy

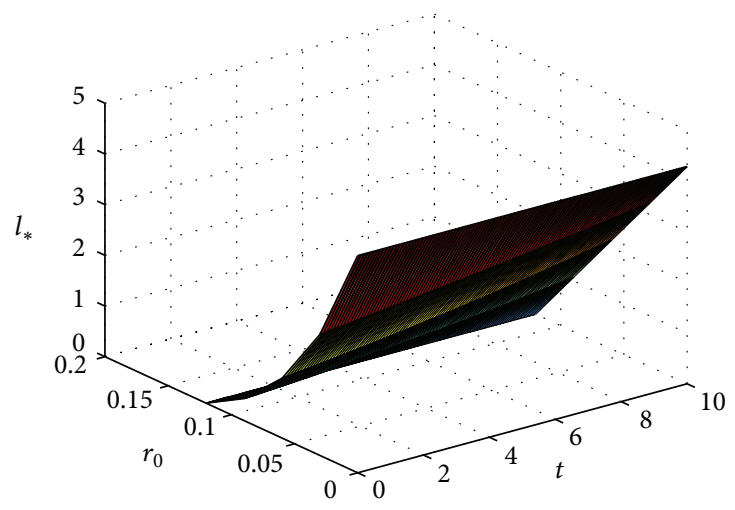

(b) The effect of $r_{0}$ and $t$ on optimal time-consistent strategy

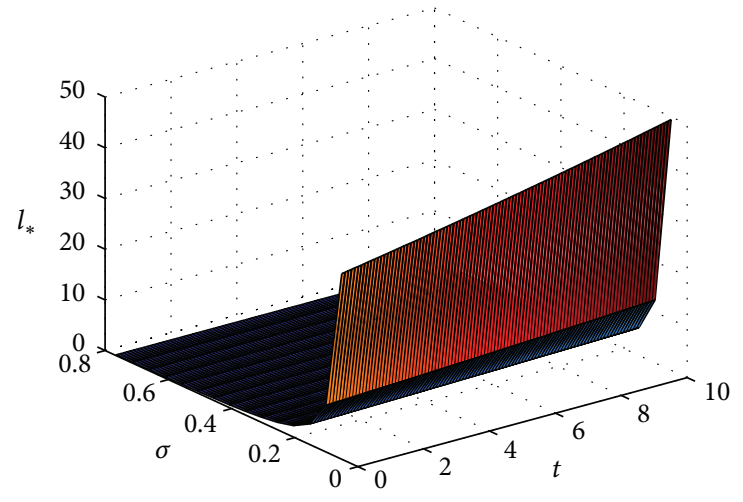

(d) The effect of $\sigma$ and $t$ on optimal time-consistent strategy

FIGURE 4: The effect of parameters on optimal time-consistent strategy.

(1) $\partial V / \partial \gamma=-\left(\left(\sigma_{0}^{2}+\lambda \sigma_{z}^{2}\right) T / 2\right)\left(\left(e^{a}-1\right) / a\right)-\left(e^{\left(\left(r_{1}-r_{0}\right)^{2} / \sigma^{2}\right) T}-\right.$ 1) $/ 2 \gamma^{2}<0$ and $\partial V / \partial \lambda=\left(\mu_{z} / r_{0}\right)\left(e^{r_{0} T}-1\right)-\left(\gamma \sigma_{z}^{2} T /\right.$ 2) $\left(\left(e^{a}-1\right) / a\right)>0(<0)$ when $\left(\mu_{z} / r_{0}\right)\left(e^{r_{0} T}-1\right)>$ $\left(\gamma \sigma_{z}^{2} T / 2\right)\left(\left(e^{a}-1\right) / a\right)\left(\left(\mu_{z} / r_{0}\right)\left(e^{r_{0} T}-1\right)<\left(\gamma \sigma_{z}^{2} T / 2\right)\right.$ $\left(\left(e^{a^{z}}-1\right) / a\right)$, which means as the coefficient risk aversion $\gamma$ increases or as the intensity of the jumps of the profit $\lambda$ decreases (increases), the optimal meanvariance utilities decrease; see Figure 3(a).

(2) $\partial V / \partial r_{1}=\left(\gamma\left(\lambda \sigma_{z}^{2}+\sigma_{0}^{2}\right)\left(r_{1}-r_{0}\right) T^{2} / \sigma^{2}\right)\left(\left(e^{a} a-\left(e^{a}-1\right)\right) /\right.$ $\left.a^{2}\right)+\left(\left(r_{1}-r_{0}\right) T / \gamma \sigma^{2}\right) e^{\left(\left(r_{1}-r_{0}\right)^{2} / \sigma^{2}\right) T}>0$ and $\partial V / \partial \sigma^{2}=$ $-\gamma\left(\lambda \sigma_{z}^{2}+\sigma_{0}^{2}\right)\left(r_{1}-r_{0}\right)^{2} T^{2} / 2 \sigma^{4} \times\left(e^{a} a-\left(e^{a}-1\right)\right) / a^{2}-\left(\left(r_{1}-\right.\right.$ $\left.\left.r_{0}\right)^{2} T / 2 \gamma \sigma^{4}\right) e^{\left(\left(r_{1}-r_{0}\right)^{2} / \sigma^{2}\right) T}<0$, which illustrates that when the appreciation rate $r_{1}$ increases or the volatility of the market's risky asset $\sigma^{2}$ decreases, the optimal mean-variance utilities increase; see Figure 3(b).

(3) $\partial V / \partial \mu_{z}=\left(\lambda / r_{0}\right)\left(e^{r_{0} T}-1\right)>0$ and $\partial V / \partial \sigma_{z}^{2}=-(\gamma \lambda T /$ 2) $\left(\left(e^{a}-1\right) / a\right)<0$, which shows that when the expectation of the size of each income $\mu_{z}$ increases or the second moment of the size of each income $\sigma_{z}^{2}$ decreases, the optimal mean-variance utilities increase; see Figure 3(c).
(4) $\partial V / \partial \sigma_{0}^{2}=-(\gamma T / 2) \times\left(\left(e^{a}-1\right) / a\right)<0$, which reveals that the value function is decreasing with respect to $\sigma_{0}^{2}$; namely, when the uncertainty of the profit increases, the optimal mean-variance utilities decrease; see Figure 3(d).

5.2. Analysis of Optimal Time-Consistent Strategy and the Equilibrium Value Function. In this subsection, we will work on numerical analysis of the time-consistent strategy and the equilibrium value function.

Firstly, we work on how the coefficients involved impact optimal time-consistent investment strategy. From (73) it is easy to see that $\partial l_{*} / \partial t=\left(r_{0}\left(r_{1}-r_{0}\right) / \gamma \sigma^{2}\right) e^{-r_{0}(T-t)}>0$, which means the company will invest more money into the risky asset as time goes by and also obtain the following findings.

(1) $\partial l_{*} / \partial \gamma=-l_{*} / \gamma<0$, which illustrates that the more the company dislikes risk, the less amount the company invests into the risky asset; see Figure 4(a).

(2) $\partial l_{*} / \partial r_{0}=-\left(\left(1+\left(r_{1}-r_{0}\right)(T-t)\right) / \gamma \sigma^{2}\right) e^{-r_{0}(T-t)}<0$, which reveals that the smaller the risk-free rate is, the more amount the company invests into the risky asset; see Figure 4(b). 


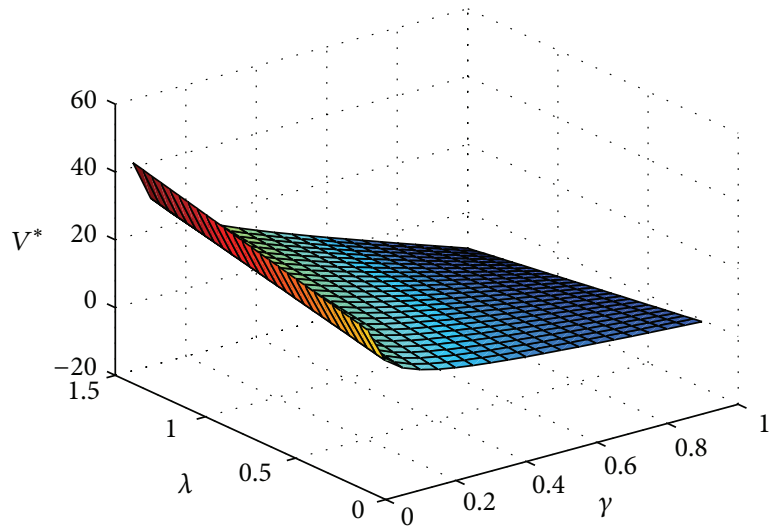

(a) The effect of $\gamma$ and $\lambda$ on the equilibrium value function

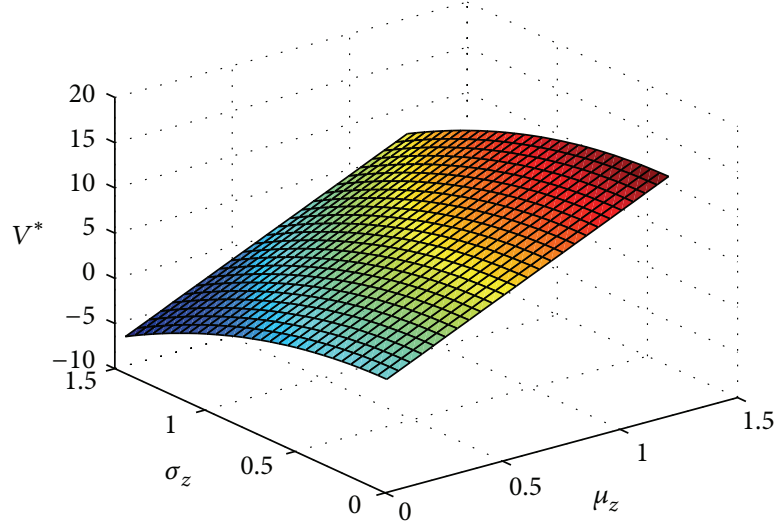

(c) The effect of $\mu_{z}$ and $\sigma_{z}$ on the equilibrium value function

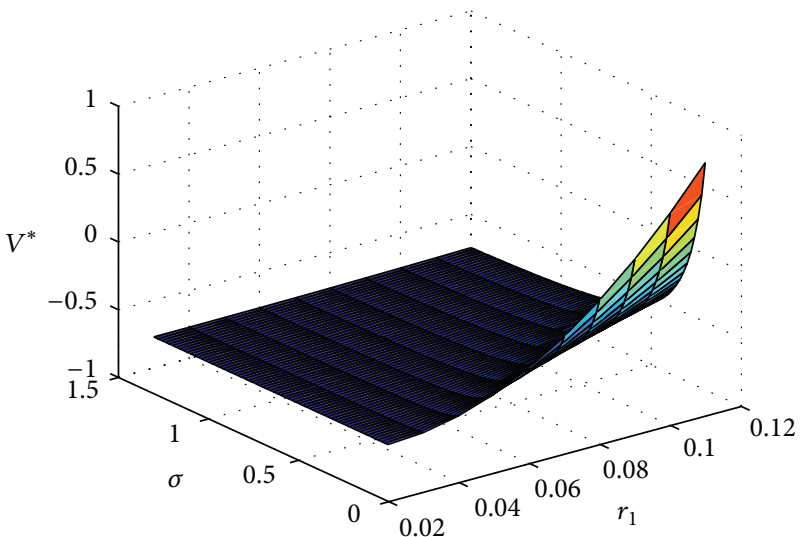

(b) The effect of $r_{1}$ and $\sigma$ on the equilibrium value function

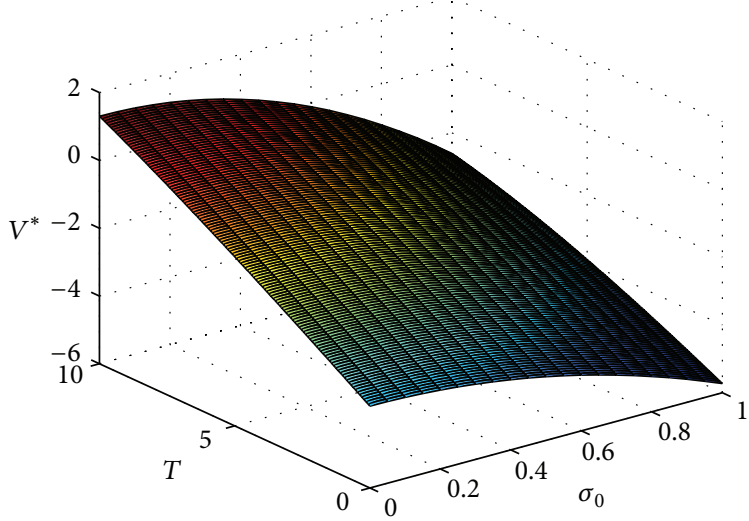

(d) The effect of $\sigma_{0}$ and $T$ on the equilibrium value function

FIGURE 5: The effect of parameters on the equilibrium value function.

(3) $\partial l_{*} / \partial r_{1}=\left(1 / \gamma \sigma^{2}\right) e^{-r_{0}(T-t)}>0$, which reveals when the appreciation rate $r_{1}$ increases, the company should invest more money into the risky asset; see Figure 4(c).

(4) $\partial l_{*} / \partial \sigma^{2}=-l_{*} / \sigma^{2}<0$, which tells that when the volatility of the risky asset increases, the company should invest more money into the risk-free asset; see Figure 4(d).

Secondly, we will show how the coefficients involved impact the value function. From (74), we can conclude the following findings:

$$
\begin{gathered}
\frac{\partial V}{\partial \gamma}=-\frac{\left(\sigma_{0}^{2}+\lambda \sigma_{z}^{2}\right)}{4 r_{0}}\left(e^{2 r_{0} T}-1\right)-\frac{1}{2 \gamma^{2}} \frac{\left(r_{1}-r_{0}\right)^{2}}{\sigma^{2}} T<0, \\
\frac{\partial V}{\partial \lambda}=\frac{\mu_{z}}{r_{0}}\left(e^{r_{0} T}-1\right)-\frac{\gamma \sigma_{z}^{2}}{4 r_{0}}\left(e^{2 r_{0} T}-1\right), \\
\frac{\partial V}{\partial r_{1}}=\frac{r_{1}-r_{0}}{\gamma \sigma^{2}} T>0, \quad \frac{\partial V}{\partial \sigma^{2}}=-\frac{\left(r_{1}-r_{0}\right)^{2} T}{2 \gamma \sigma^{4}}<0,
\end{gathered}
$$

$$
\begin{gathered}
\frac{\partial V}{\partial \mu_{z}}=\frac{\lambda}{r_{0}}\left(e^{r_{0} T}-1\right)>0, \quad \frac{\partial V}{\partial \sigma_{z}^{2}}=-\frac{\gamma \lambda}{4 r_{0}}\left(e^{2 r_{0} T}-1\right)<0, \\
\frac{\partial V}{\partial \sigma_{0}^{2}}=-\frac{\gamma}{4 r_{0}}\left(e^{2 r_{0} T}-1\right)<0 .
\end{gathered}
$$

Figure 5 shows that how the coefficients involved impact the equilibrium value function. The parameters $\gamma, \lambda, r_{1}, \sigma^{2}, \mu_{z}, \sigma_{z}^{2}$, and $\sigma_{0}^{2}$ have the similar effect on the equilibrium value function as their effect on the value function with precommitment discussed in Section 5.1.

5.3. Comparisons between the Precommitted Strategy and the Time-Consistent Strategy. In this subsection, we compare the optimal investment strategy, the corresponding value function, and the efficient frontier under the precommitted framework with the ones under the time-consistent framework.

Firstly, we compare the precommitted strategy with the time-consistent strategy. The time-consistent strategy is time deterministic but the precommitted strategy depends on the current wealth. We also assume that $\{N(t)\}_{t \geq 0}$ is a Poisson process with intensity $\lambda$ and $Z_{j}(j=1,2,3 \ldots)$ 


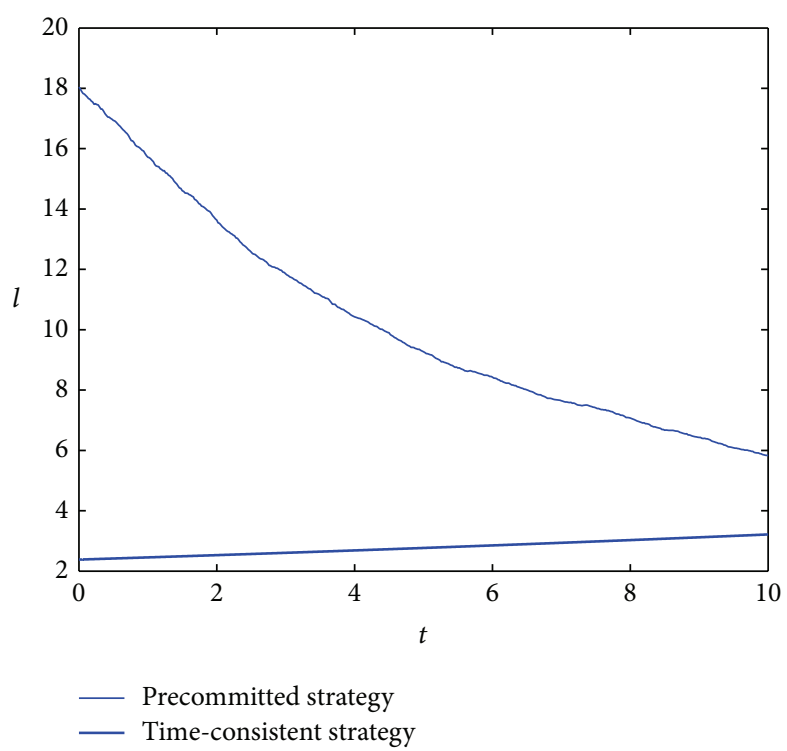

(a) The comparison of optimal strategies

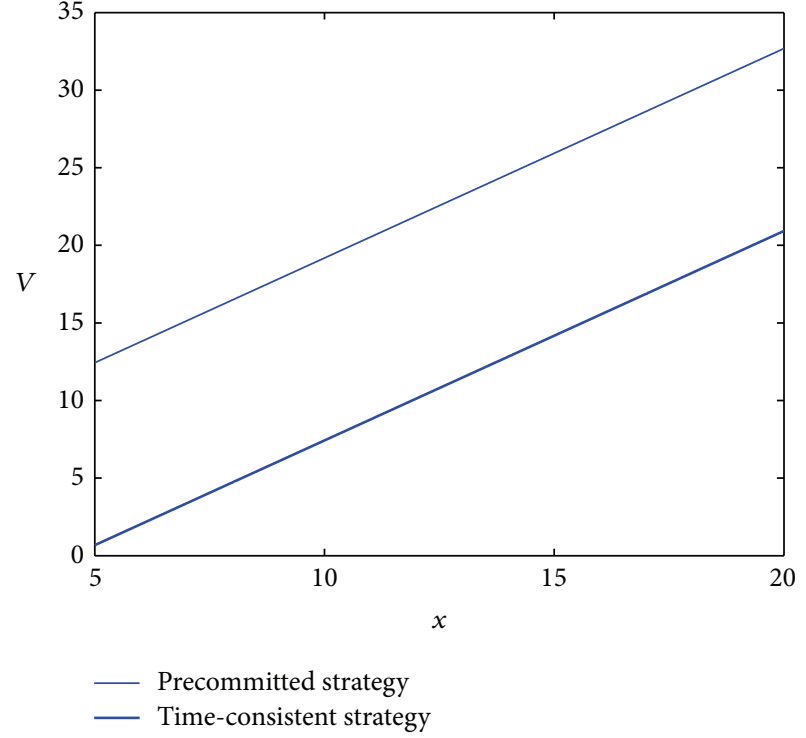

(b) The comparison of the value functions

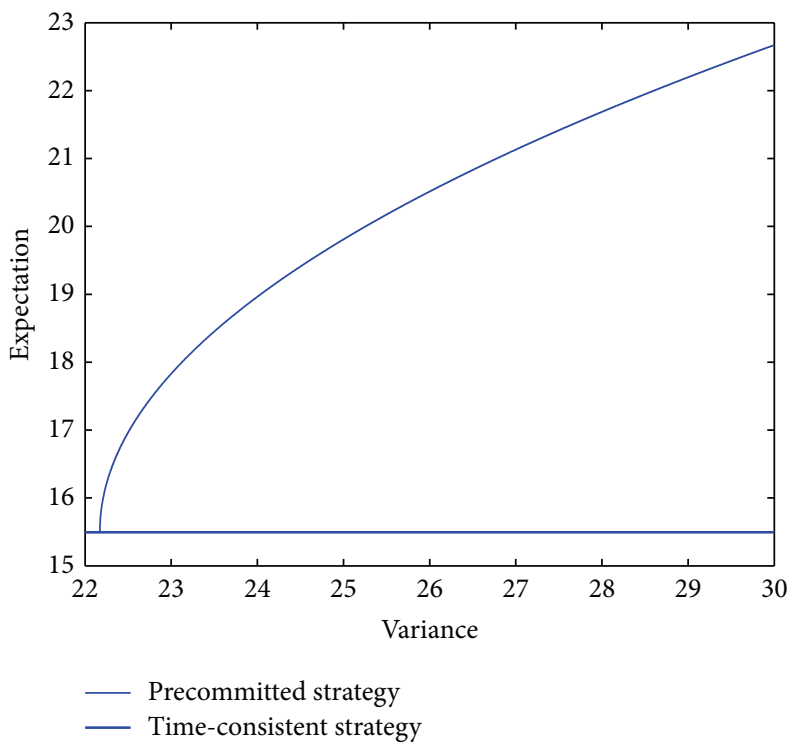

(c) The comparison of different efficient frontiers

FIGURE 6: The comparisons between the precommitted strategy and the time-consistent strategy.

are double exponentially distributed with their probability density function $f_{z}(z)=p \times \alpha e^{-\alpha z} I_{z>0}+(1-p) \times \beta e^{\beta z} I_{z<0}$. Here, fix the parameter $\lambda=1.25, p=0.4, \alpha=0.2$, and $\beta=0.8$. We investigate the precommitted investment strategy by exploiting Monte Carlo Methods. We simulate 2000 tracks of the precommitted investment strategy and calculate the average of 2000 tracks. Figure 6(a) illustrates that the average of the precommitted investment strategy is bigger than the time-consistent investment strategy which means that the company with the time-consistent strategy makes a more conservative investment than the one with the precommitted strategy on the long term.

Secondly, we compare the optimal value function $V(x)$ with the equilibrium value function $V^{*}(x)$. By a simple calculation, we can conclude that $V(t)>V^{*}(x)$ which means that the company with the time-consistent strategy has to give up the chance to attain greater current utility in order to ensure a consistent return for the whole time horizon as is illustrated in Figure 6(b).

Thirdly, we compare the efficient frontiers derived from two different perspectives. From (46) and (75) we can see that the efficient frontiers are no longer straight lines no matter in each perspective. The efficient frontier under the timeconsistent strategy is never above the efficient frontier under the precommitted strategy as is illustrated by Figure 6(c).

It seems to be true that the precommitted strategy is prior to the time-consistent strategy from the comparison of the value functions and the efficient frontiers, but we 
cannot conclude that the precommitted strategy is better than that from the game theoretical framework. Because the latter strategy is time-consistent and it can make the company ensure a consistent return for the whole time horizon. Meanwhile, the precommitted strategy is a global optimal strategy which only can make the company's mean-variance utilities maximized at $t=0$. Furthermore, the time-consistent strategy is suboptimal strategy for all $t \geq 0$ and the investment problem for all $t \geq 0$ can be viewed as a noncooperative game with one player for each time t. The time-consistent strategy can make this entire system equilibrium and it is suboptimal for the player $t$. Correspondingly, the precommitted strategy is a global optimal strategy for player $t=0$ and it cannot make the entire system equilibrium.

\section{Conclusion}

In this paper, we have investigated the optimal investment strategy for a general risk model under mean-variance criterion. The precommitted strategy is derived by the lagrange method and the time-consistent strategy is also calculated via the approach based on the time-consistent equilibrium controls. In the end, we theoretically and numerically provide the effect of the parameters on the optimal investment strategies and the corresponding value functions. The value function and the efficient frontier under the precommitted strategy are prior to the ones under the time-consistent strategy, we cannot conclude that the precommitted strategy is better than that from game theoretical framework, because the company under the time-consistent strategy has to give up the better current utility in order to keep a consistent satisfaction over the whole time horizon. Meanwhile, the precommitted strategy is a global optimal strategy and it only can make the company's mean-variance utilities maximized at initial time $t=0$.

\section{Conflict of Interests}

The authors declare that there is no conflict of interests regarding the publication of this paper.

\section{Acknowledgments}

This research is supported by the National Natural Science Foundation of China (Grant nos. 11201335 and 71071111) and the Research Project of the Social Science and Humanity on Young Fund of the Ministry of Education (Grant no. 11YJC910007). The authors would like to thank anonymous reviewers for very helpful suggestions which improved this paper greatly.

\section{References}

[1] H. Markowitz, "Portfolio selection," Journal of Finance, vol. 7, pp. 77-98, 1952.

[2] X. Y. Zhou and D. Li, "Continuous-time mean-variance portfolio selection: a stochastic LQ framework," Applied Mathematics and Optimization, vol. 42, no. 1, pp. 19-33, 2000.
[3] D. Li and W.-L. Ng, "Optimal dynamic portfolio selection: multiperiod mean-variance formulation," Mathematical Finance, vol. 10, no. 3, pp. 387-406, 2000.

[4] T. Björk and A. Murgoci, "A general theory of Markovian time inconsistent stochastic control problems," Working Paper, Stockholm School of Economics, 2010.

[5] I. Ekeland and A. Lazrak, "Being serious about noncommitment:subgame perfect equilibrium in continuous time," http://arxiv.org/abs/math/0604264.

[6] E. M. Kryger and M. Steffensen, "Some solvable portfolio problems with quadratic and collective objectives," Working Paper, 2010.

[7] N. Bäuerle, "Benchmark and mean-variance problems for insurers," Mathematical Methods of Operations Research, vol. 62, no. 1, pp. 159-165, 2005.

[8] L. Bai and H. Zhang, "Dynamic mean-variance problem with constrained risk control for the insurers," Mathematical Methods of Operations Research, vol. 68, no. 1, pp. 181-205, 2008.

[9] Z. F. Li, Y. Zeng, and Y. Z. Lai, "Optimal time-consistent investment and reinsurance strategies for insurers under Heston's SV model," Insurance: Mathematics \& Economics, vol. 51, no. 1, pp. 191-203, 2012.

[10] Y. Zeng, Z. F. Li, and Y. Z. Lai, "Time-consistent investment and reinsurance strategies for mean-variance insurers with jumps," Insurance: Mathematics \& Economics, vol. 52, no. 3, pp. 498-507, 2013.

[11] W. H. Fleming and H. M. Soner, Controlled Markov Processes and Viscosity Solutions, Springer, New York, NY, USA, 2006. 


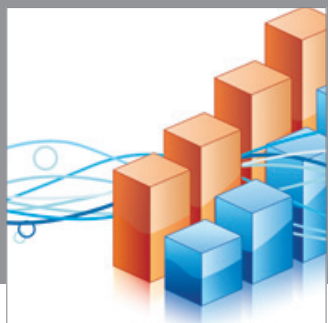

Advances in

Operations Research

mansans

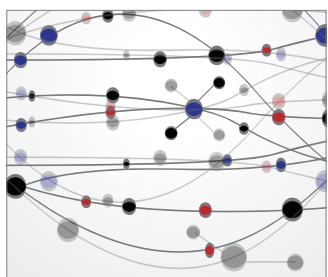

The Scientific World Journal
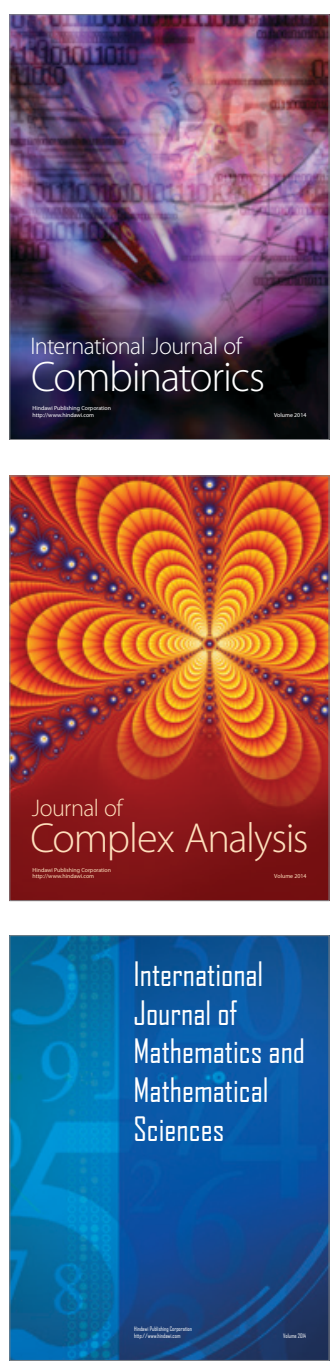
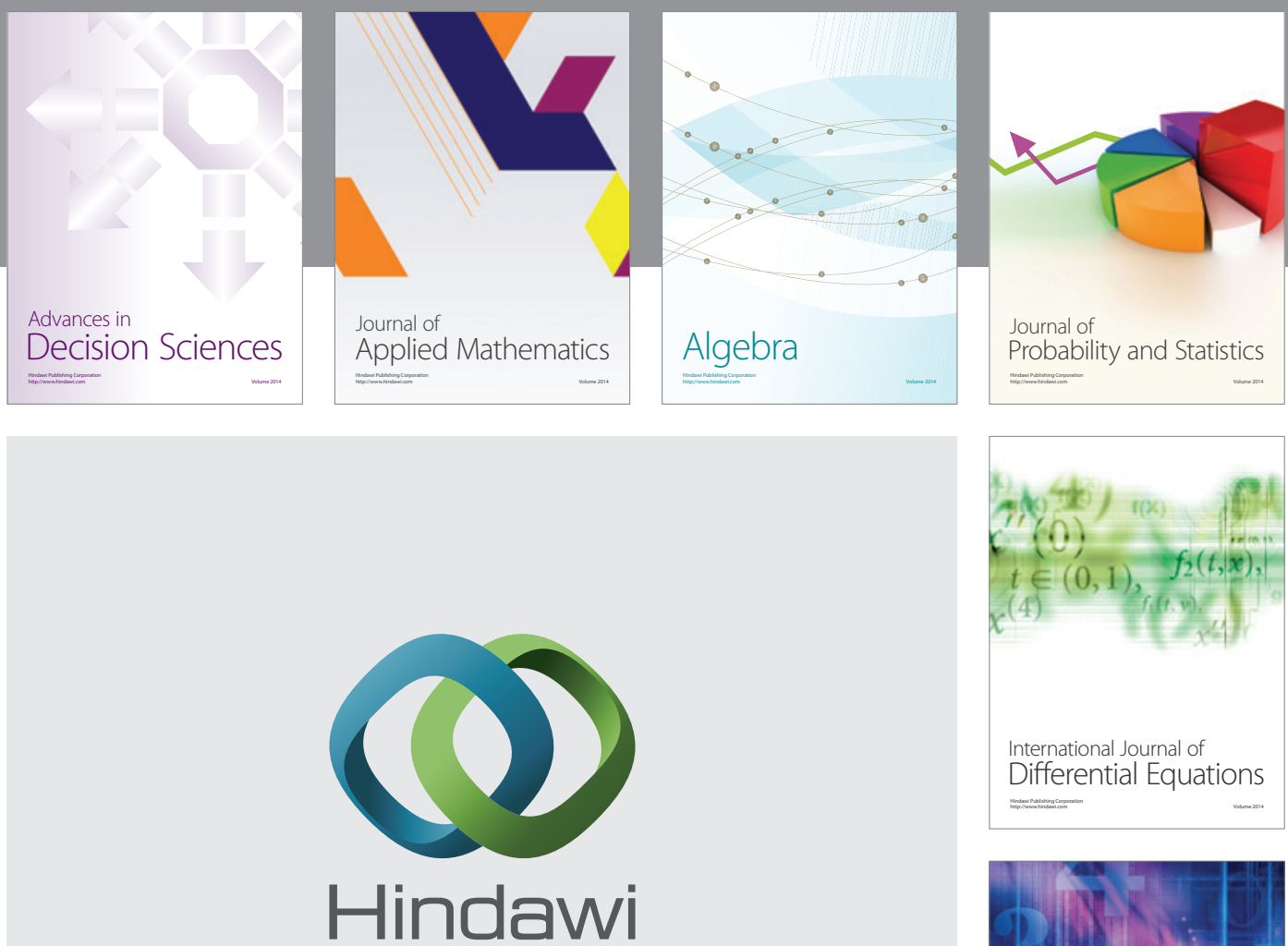

Submit your manuscripts at http://www.hindawi.com
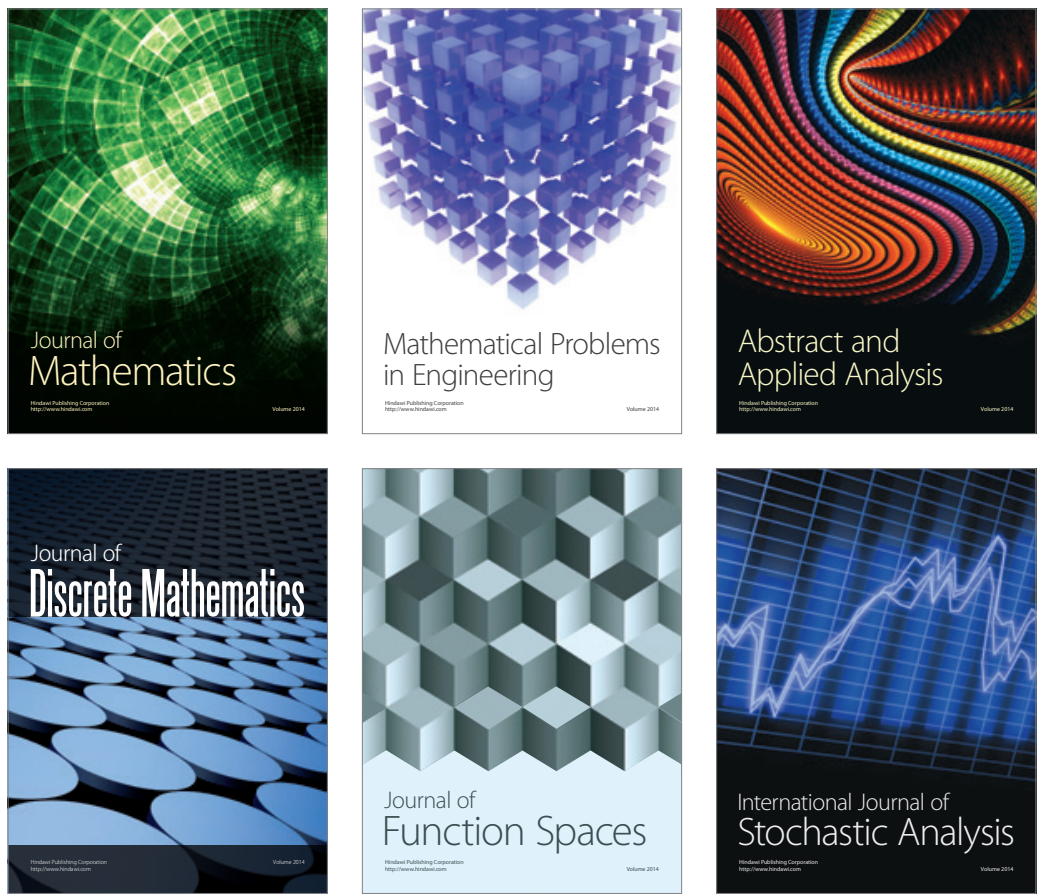

Journal of

Function Spaces

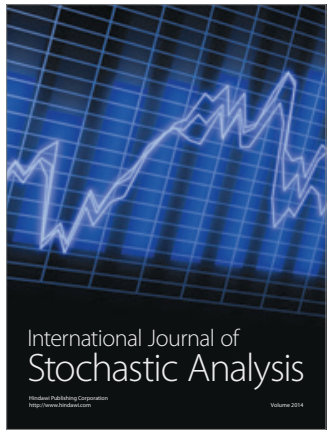

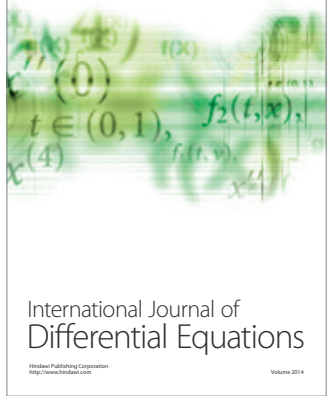
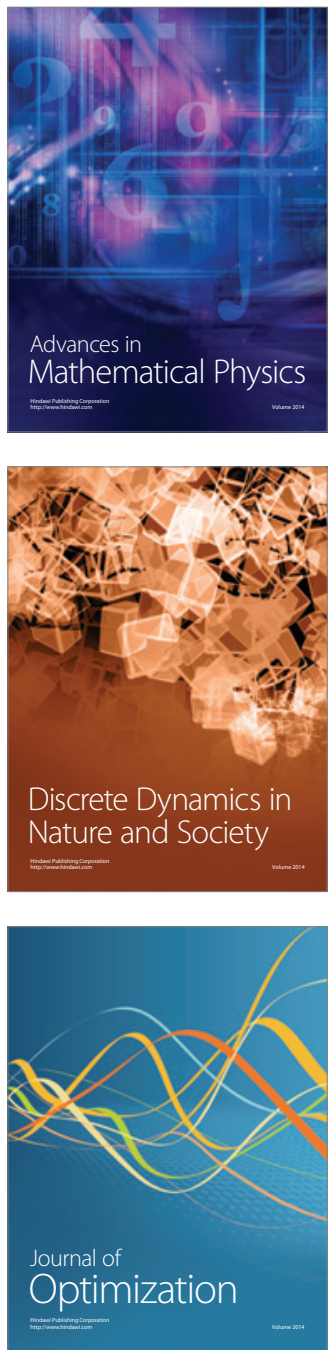